\title{
Checklist de Cladocera de água doce do Estado de São Paulo
}

\author{
Odete Rocha ${ }^{1,4}$, Maria José Santos-Wisniewski² \& Takako Matsumura-Tundisi ${ }^{3}$ \\ ${ }^{1}$ Departamento de Ecologia e Biologia Evolutiva, Universidade Federal de São Carlos - UFSCar, \\ Rod. Washington Luis, Km 235, CP 676, CEP 13565-605, São Carlos, SP, Brasil \\ ${ }^{2}$ Instituto de Ciências da Natureza, Universidade Federal de Alfenas - UNIFAL, \\ Rua Gabriel Monteiro da Silva, 714, Centro, CEP 37130-000, Alfenas, MG, Brasil, \\ e-mail: czw@uol.com.br \\ ${ }^{3}$ Instituto Internacional de Ecologia, Rua Bento Carlos, 750, Centro, \\ CEP 13560-660, São Carlos, SP, Brasil \\ ${ }^{4}$ Autor para correspondência: Odete Rocha,e-mail: doro@ufscar.br
}

ROCHA, O., SANTOS-WISNIEWSKI, M.J. \& MATSUMURA-TUNDISI, T. Checklist of fresh-water Cladocera from São Paulo State, Brazil. Biota Neotrop. 11(1a): http://www.biotaneotropica.org.br/v11n1a/ en/abstract?inventory+bn0271101a2011.

\begin{abstract}
In the present work an updated checklist of the species of Cladocera in the state of São Paulo is presented, based on previous check-list and a review of the recent studies which include in majority the studies developed within the BIOTA/FAPESP Program. Species inventory performed ten years ago revealed the occurrence of 112 species in Brazil and 84 species in the State of São Paulo. The present review shows the occurrence of 96 species of this group in the state of São Paulo, representing a 15\% increase in the species richness. Among the 300 water bodies sampled in 23 units water resource management units (UGHRI) of São Paulo State within the scope of the BIOTA/FAPESP Program, the highest richness of Cladocera species was found in Mogi-Guaçu and Aguapeí units with 27 species recorded in each. Among the advances reached by the BIOTA/FAPESP Program it could be emphasized the wide geographic covering and the number of species recorded, with 12 new occurrences of native species and 2 exotic species as well. This study evidenced that still there are important gaps in the knowledge, particularly regarding the taxonomy, since many species regarded as cosmopolitan may be a complex of many species requiring taxonomical reviews and ecological studies of the species. It is believed that with the continuity of the studies, the richness of species of Cladocera can increase considerably.
\end{abstract} Keywords: fresh-water Cladocera, biodiversity of the State of São Paulo, BIOTA/FAPESP Program.

Number of species: In the world: 620, In Brazil: 150, Estimated in São Paulo State: 120.

ROCHA, O., SANTOS-WISNIEWSKI, M.J. \& MATSUMURA-TUNDISI, T. Checklist dos Cladocera de água doce do Estado de São Paulo, Brasil. Biota Neotrop. 11(1a): http://www.biotaneotropica.org.br/v11n1a/ pt/abstract?inventory+bn0271101a2011.

Resumo: Neste trabalho é apresentada uma lista atualizada das espécies de Cladocera do Estado de São Paulo com base em levantamentos anteriores e em revisão da literatura recente, a qual inclui em grande parte os estudos oriundos do Programa Biota FAPESP. Levantamentos realizados há uma década apontaram a ocorrência de 112 espécies de Cladocera no Brasil e 84 para o Estado de São Paulo. A presente revisão evidencia a ocorrência de 96 espécies deste grupo no Estado de São Paulo, o que representa um acréscimo de $15 \%$ na riqueza de espécies. Dentre os 300 corpos de água amostrados em 23 unidades de gerenciamento dos recursos hídricos do Estado de São Paulo, no âmbito do Programa BIOTA/FAPESP, as unidades Mogi-Guaçu e Aguapeí são aquelas com maior riqueza de espécies, tendo sido registradas 27 espécies de Cladocera em cada. Dentre os avanços obtidos pelo Programa BIOTA/FAPESP destacaram-se a ampla cobertura geográfica e o registro das novas ocorrências de espécies, das quais 12 são espécies nativas e 2 são espécies exóticas. Este estudo evidenciou que ainda existem importantes lacunas no conhecimento, especialmente em relação à taxonomia, pois muitas espécies que foram consideradas cosmopolitas são provavelmente um complexo de espécies, sendo necessárias revisões taxonômicas detalhadas acopladas a estudos ecológicos das espécies. Acredita-se que com a continuidade destes estudos a riqueza de espécies de Cladocera poderá aumentar consideravelmente.

Palavras-chave: Cladocera de água doce, biota paulista, Programa BIOTA/FAPESP.

Número de espécies: No mundo: 620, No Brasil: 150, Estimadas no Estado de São Paulo: 120. 


\section{Introdução}

Os Cladocera constituem um dos mais representativos componentes do plâncton de água doce, onde são importantes nas redes tróficas, principalmente de hábito herbívoro filtrador, desempenhando o papel de consumidores primários. Anteriormente considerados uma Ordem da Classe Crustacea e Sub-Classe Branchiopoda, eles foram recentemente sujeitos a revisões taxonômicas. Fryer (1987, 1995) e Paggi (1995) sugerem que eles não constituiriam um agrupamento natural, sendo provavelmente um grupo polifilético, cujas semelhanças seriam mais o reflexo de processos de convergência. No entanto, Forró et al. (2008) consideram se tratar de um grupo monofilético e anciente, de origem paleozóica. Os Cladocera estão atualmente reclassificados em quatro novas ordens: Anomopoda, Ctenopoda, Onychopoda e Haplopoda (Fryer 1987). Os Haplopoda e Onychopoda de água doce só ocorrem em águas continentais da região Holártica (América do Norte e parte da Eurásia). Os Haplopoda da família Leptodoridae, de hábito predatório, não ocorrem nos trópicos (Korinek 2002), estando assim ausentes no Brasil e no Estado de São Paulo. Por outro lado, representantes das Ordens Ctenopoda e Anomopoda que englobam a maioria das espécies de Cladocera distribuem-se por todos os continentes (Frey 1987b, 1995). A maior parte dos Onychopoda (famílias Podonidae, Polyphemidae, e Cercopagidae) estão restritos aos ambientes estuarinos e marinhos. A maioria das espécies de Cladocera pertence, portanto, às ordens: Ctenopoda (famílias Sididae e Holopedidae) e Anomopoda (famílias: Macrothricidae, Ilyocryptidae; Bosminidae, Daphnidae, Moinidae, e Chydoridae) Paggi (1995). Antes alocadas na sub-ordem Calyptomera, as duas primeiras famílias (Macrothricidae e Chydoridae) foram reclassificadas em uma nova sub-ordem, Radopoda (Dumont \& Silva-Briano 1998).

Os Cladocera ocupam uma grande variedade de habitats de água doce, incluindo desde pequenos volumes de água contidos em fitotelmata e em cavidades de troncos de plantas terrestres, pequenas poças e pântanos até os corpos de água maiores, onde atingem maior diversificação como nas lagoas, lagos, canais e reservatórios. Embora ocorram preferencialmente em ambientes lênticos, são também habitantes de riachos, córregos e rios, nestes ocupando preferencialmente os remansos, de fluxo mais reduzido.

Os cladóceros estão distribuídos pelos vários continentes, inclusive em regiões circumpolares (Brooks 1959). A idéia do cosmopolitismo de muitas espécies resultou de identificações feitas com base na análise morfológica superficial e descrições incompletas de espécies do Hemisfério Norte. Este conceito baseou-se também no fato de que os cladóceros produzem ovos de resistência, os quais podem ser transportados passivamente de um corpo d'água a outro por diversos animais, principalmente por aves, ou mesmo pelo vento (Frey 1982). Entretanto, sabe-se que algumas espécies são restritas a determinados climas, como por exemplo o tropical, não ocorrendo em regiões temperadas frias (Green 1981). Isso significa que mesmo na ausência de barreiras físicas importantes, a maioria das espécies tende a não se adaptar nos locais onde foram introduzidas (Frey 1987a). Neste caso os ovos de resistência adquirem papel relevante para a manutenção da variabilidade genética nas populações.

Apesar do cosmopolitismo ser amplamente questionado (Frey 1982, 1987, Forró et al. 2008) as dificuldades de revisão taxonômica são ainda muito grandes pois dependem da disponibilidade de material abundante, seja preservado para melhores descrições morfológicas, seja vivo para a realização de estudos ecológicos e análises genéticomoleculares específicas. Há, além disso, a necessidade da reunião de expertises nos campos da taxonomia, da ecologia e da genética molecular.
A ampla ocorrência dos Cladocera e a facilidade no seu cultivo viabilizam sua utilização em estudos laboratoriais e consequente aumento do conhecimento sobre o grupo. Deste modo várias pesquisas sobre a biologia das espécies e a utilização destas na realização de bioensaios para o controle da qualidade do ambiente tem sido desenvolvidas em condições padronizadas (Guntzel et al. 2003, Castilho 2005, Santos-Wisniewski et al., 2006, Nogueira et al., 2005, Freitas \& Rocha, 2006, Rosa 2008.).

No mundo, cerca de 620 espécies são conhecidas atualmente, contudo, estima-se que se fossem resolvidos os casos de falso cosmopolitismo e de espécies crípticas (complexo de espécies) este número poderia duplicar (Forró et al. 2008). Cerca 150 espécies tinham ocorrência registrada no Brasil até o final do século XX (Rocha \& Guntzel 1999), contudo parte daqueles registros eram na realidade sinonímias, as quais foram sendo corrigidas, de forma que apesar dos novos registros, o número absoluto de espécies de Chydoridae não aumentou se comparadas as listas apresentadas neste último trabalho referido e a presente síntese. No entanto, considerando os novos registros ocorridos ao longo da última década (ElmoorLoureiro 2004, Lansac-Tôha et al. 2009, Sinev \& Elmoor-Loureiro 2010) e o registro da invasão por espécies exóticas (Zanata et al. 2003) este número tem aumentado.

No Estado de São Paulo há registros de espécies pertencentes a todas as sete famílias da Ordem Anomopoda (Sididae, Moinidae, Daphnidae, Bosminidae, Macrothricidade Ilyocryptidae e Chydoridae). Dentre estas, as famílias Daphnidae, Bosminidae, Sididae e Moinidae são importantes componentes do plâncton limnético, com ocorrência em um grande número e variedade de corpos de água. As espécies destas famílias são bem conhecidas e é provável que poucas espécies não tenham ainda sido registradas. Por outro lado, as espécies das famílias e Chydoridae, Macrothricidae e Ilyocryptidae, tipicamente associadas a substratos, principalmente às macrófitas, com ocorrência principalmente na região litorânea dos corpos de água tem amplas possibilidades de ampliação no número de espécies, principalmente pela revisão das espécies cosmopolitas.

\section{Metodologia}

Para este trabalho baseou-se na revisão de literatura realizada no início do Programa Biota/FAPESP (Rocha \& Guntzel 1999) e na revisão atual sobre as publicações em periódicos nos últimos 10 anos e na compilação de registros de espécies contidos em teses, dissertações, e monografias de conclusão de cursos, além de material disponível em sites confiáveis na internet.

\section{Resultados e Discussão}

\section{Comentários sobre a lista das espécies do Estado de São Paulo}

Na Tabela 1 é apresentada a lista das espécies registradas no Estado de São Paulo, com base nos dados obtidos no Programa BIOTA/FAPESP sobre o zooplâncton das águas doces e na revisão de literatura efetuada.

No mundo, são conhecidas atualmente cerca de 620 espécies, contudo, acredita-se que se resolvidos os casos de falso cosmopolitismo e de espécies crípticas este número poderia duplicar (Forró et al. 2008). Até o final da década passada cerca de 112 espécies tinham ocorrência registrada no Brasil, e 84 no Estado de São Paulo (Rocha \& Guntzel 1999). Contudo, parte dos registros no Estado de São Paulo referia-se a sinonímias, as quais foram sendo corrigidas, de forma que apesar de novos registros, o número absoluto de espécies de Cladocera não aumentou muito se comparadas as listas apresentadas 


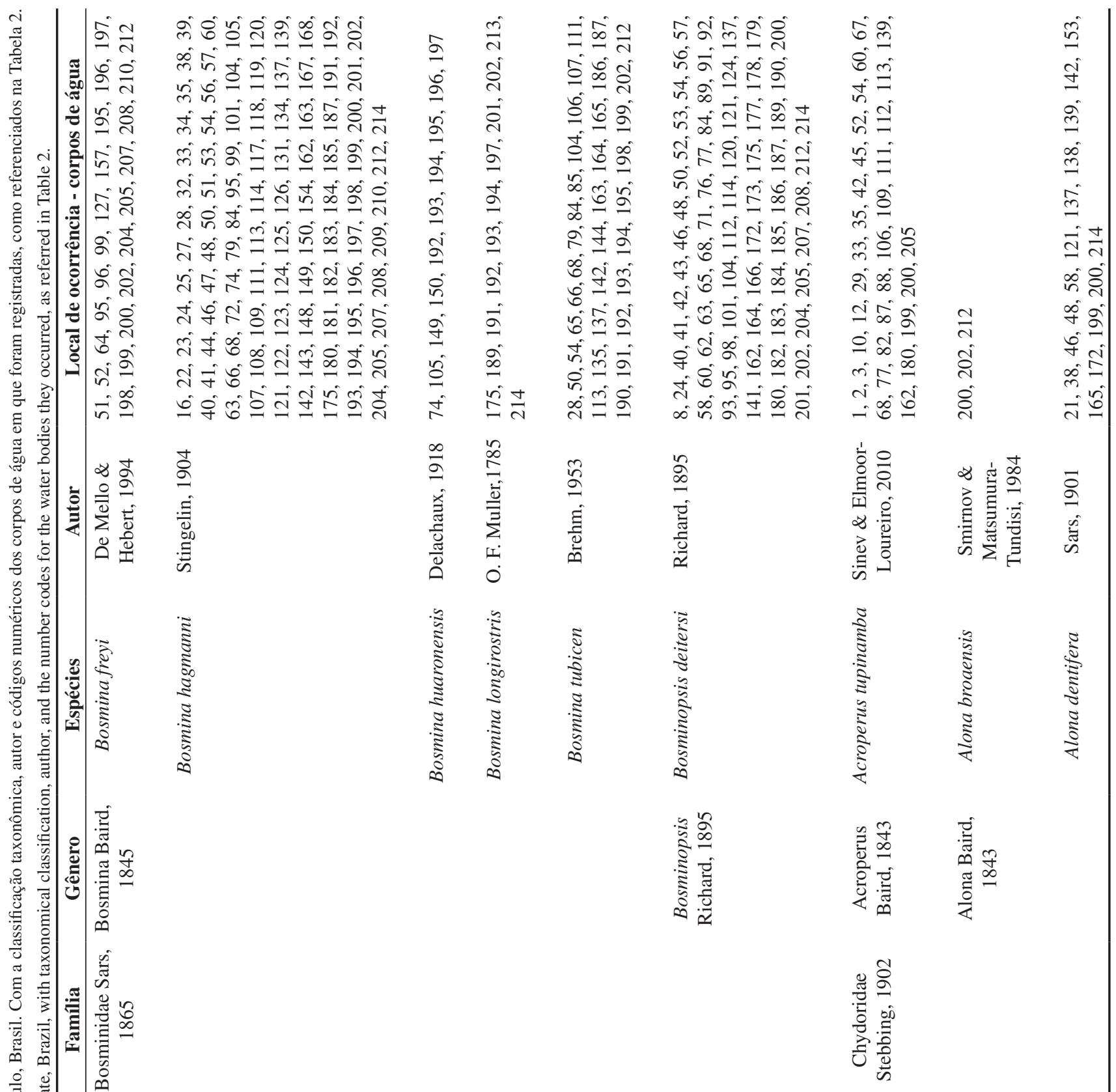


Rocha, O. et al.

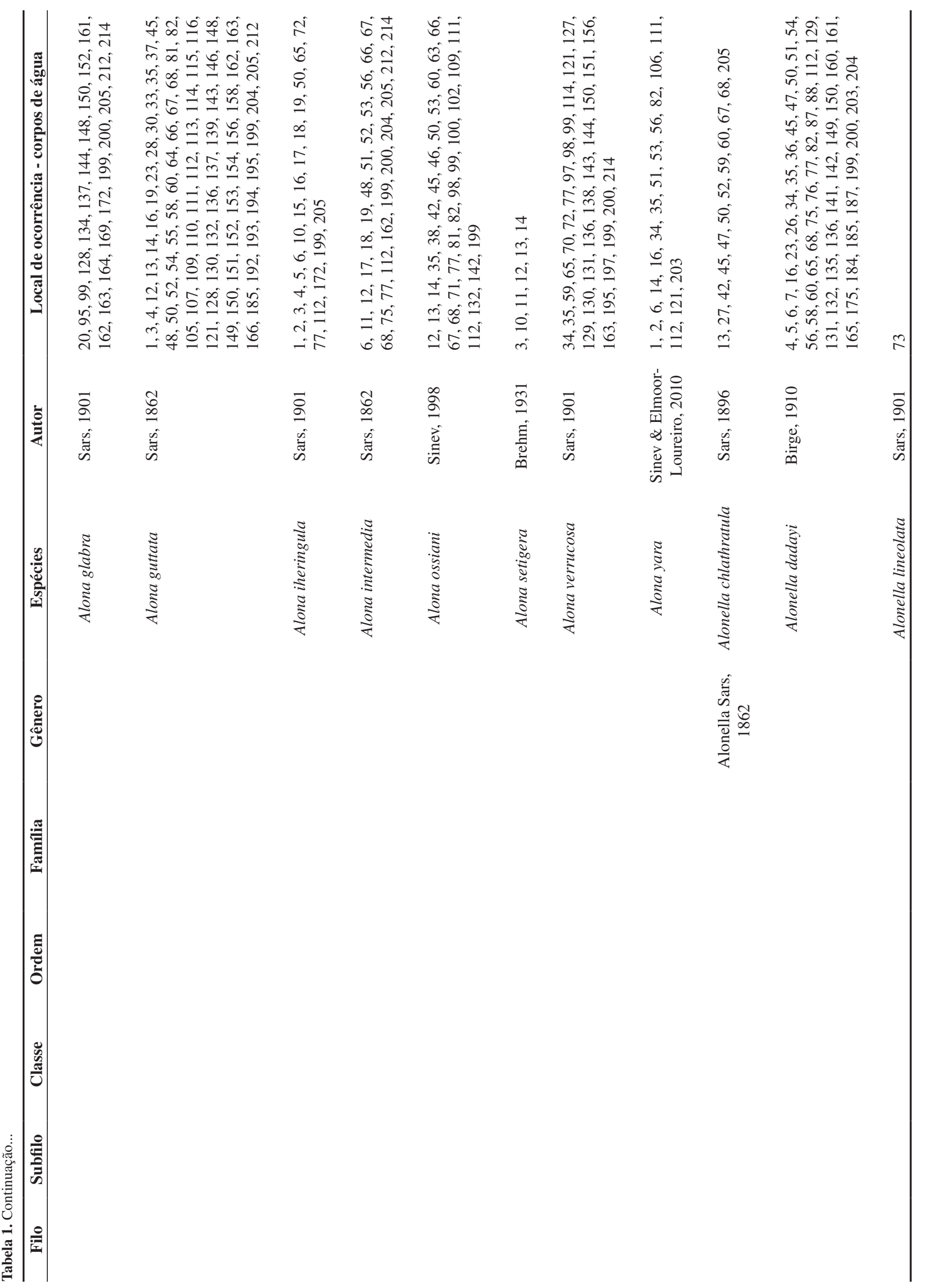




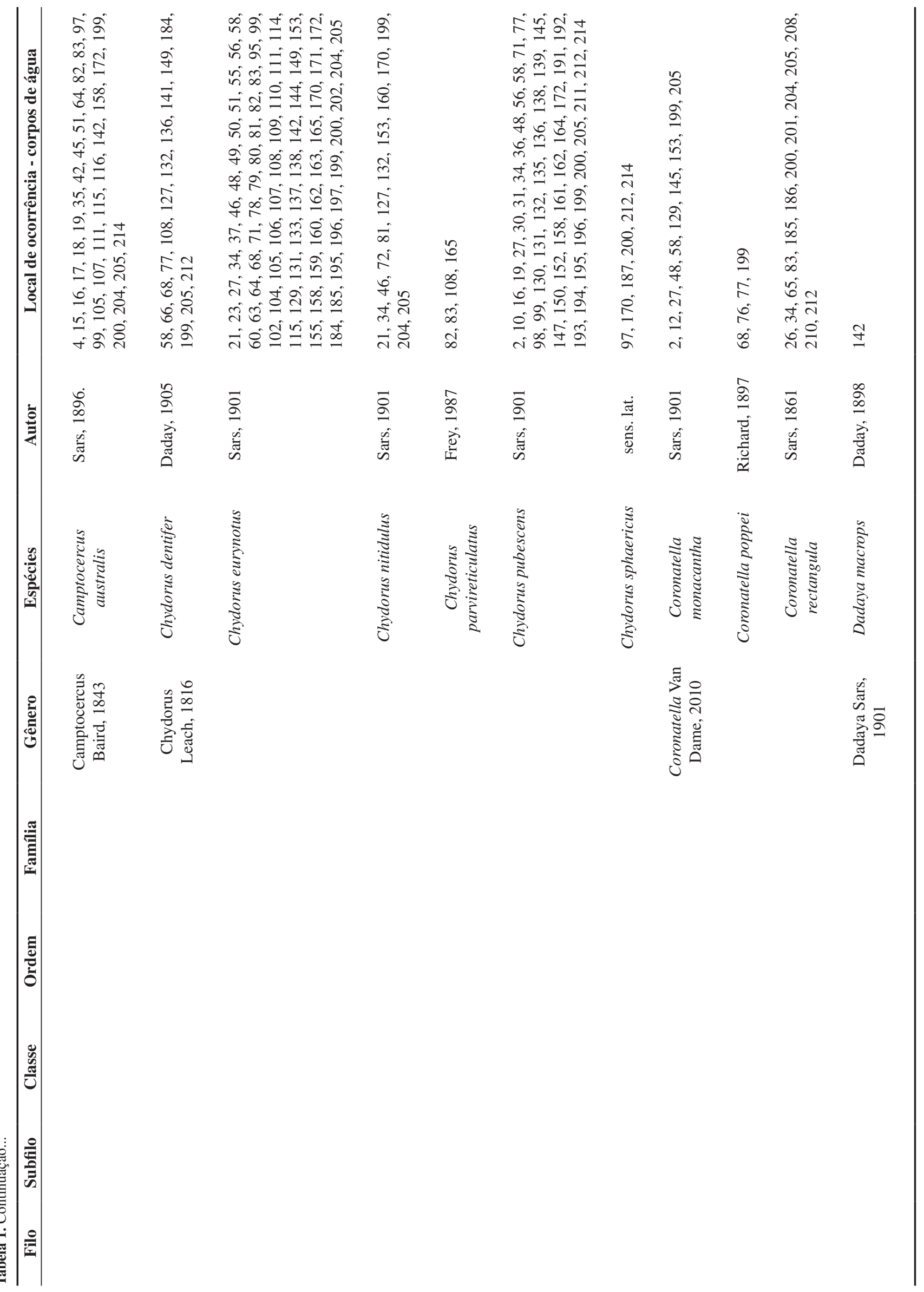


Rocha, O. et al.

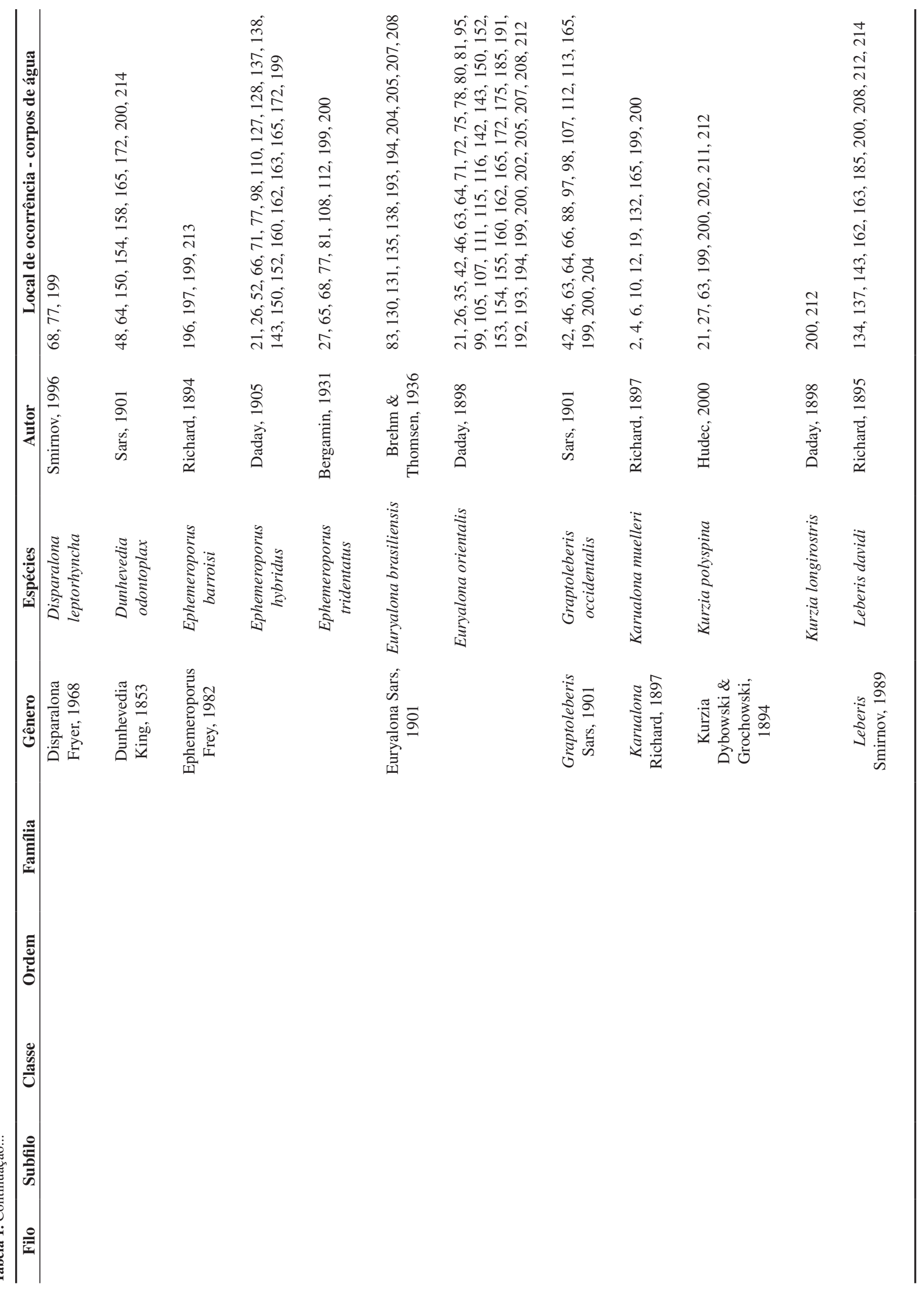


Checklist de Cladocera de água doce do Estado de São Paulo

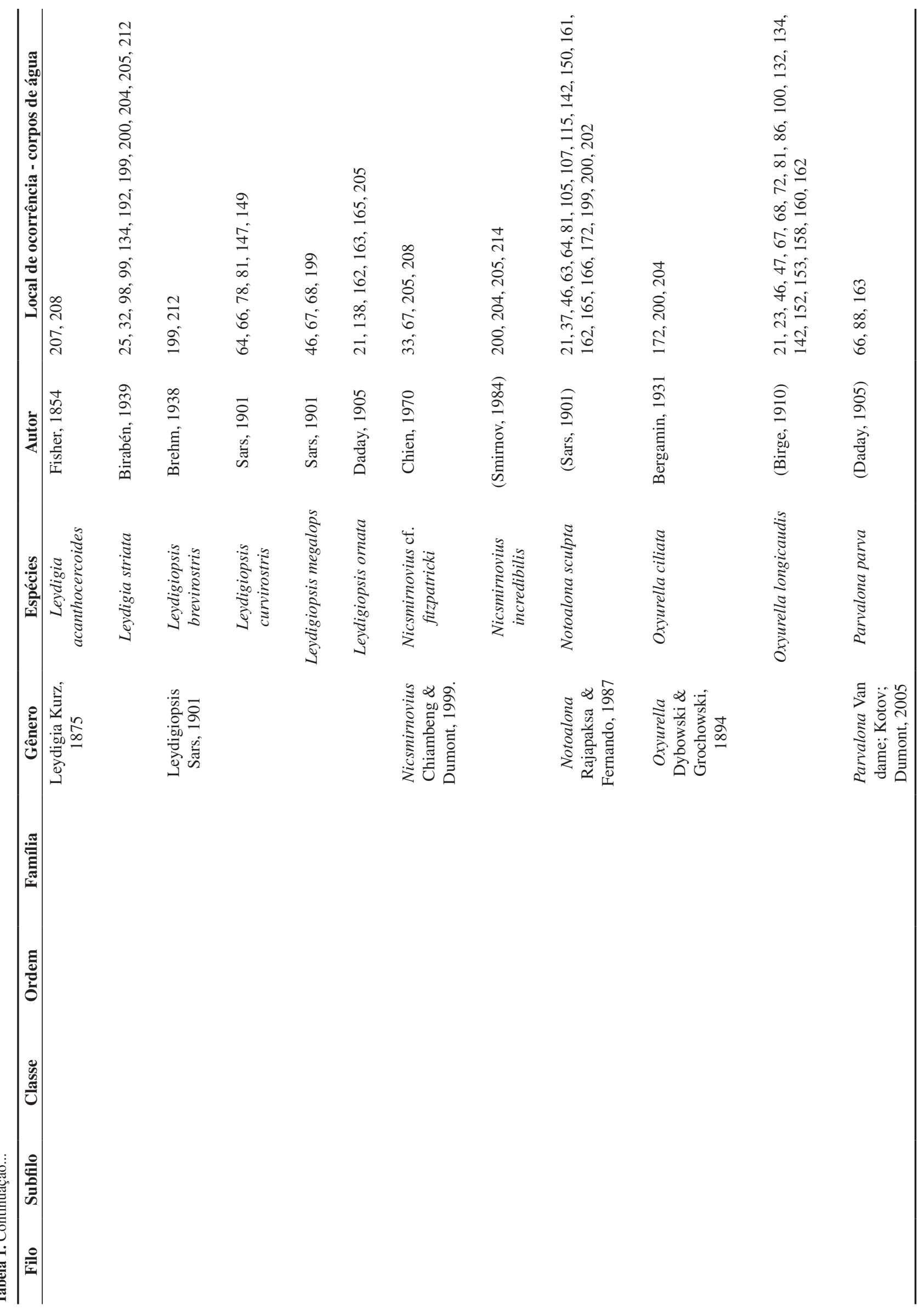


Rocha, O. et al.

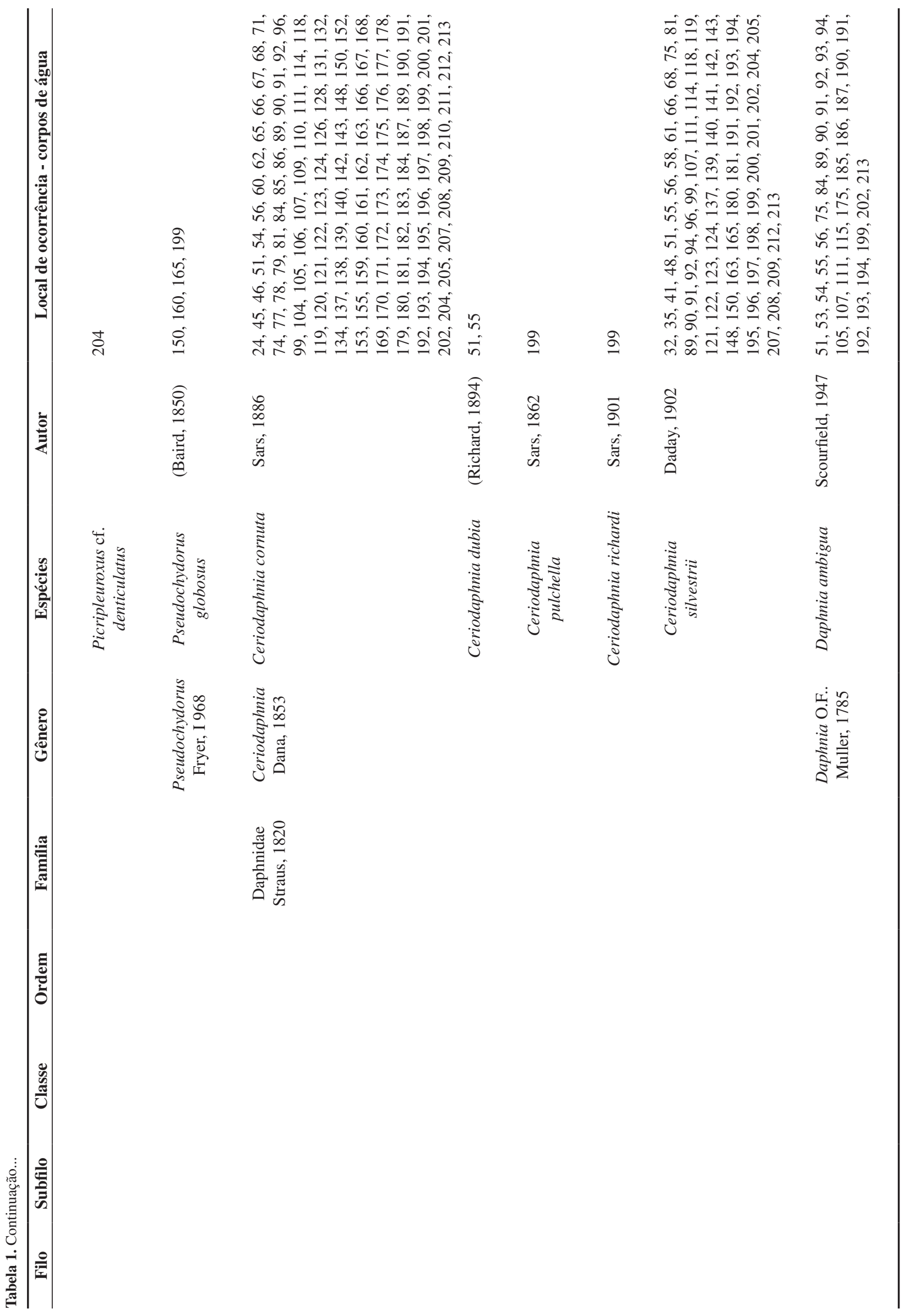


Checklist de Cladocera de água doce do Estado de São Paulo

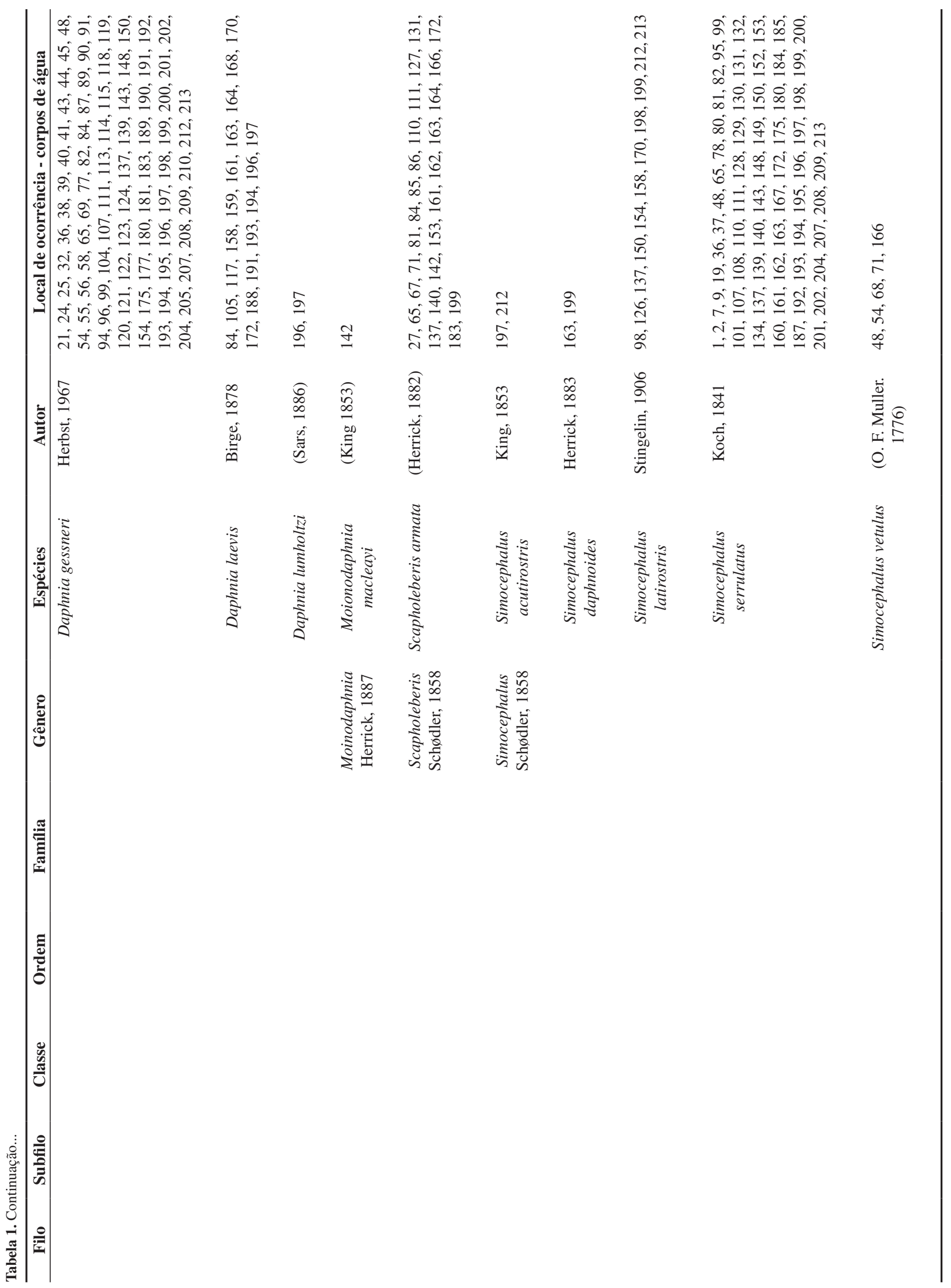


Rocha, O. et al.

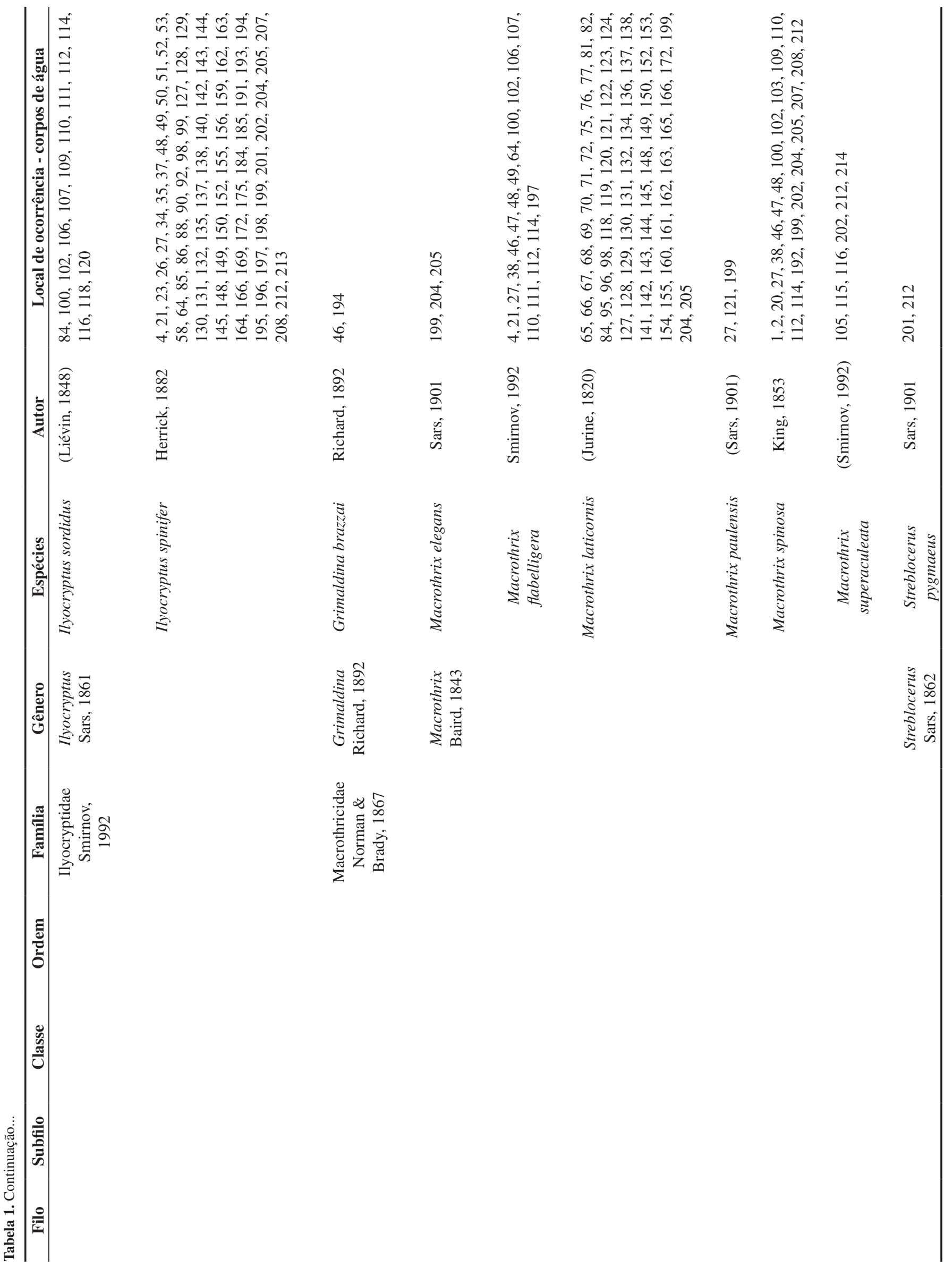




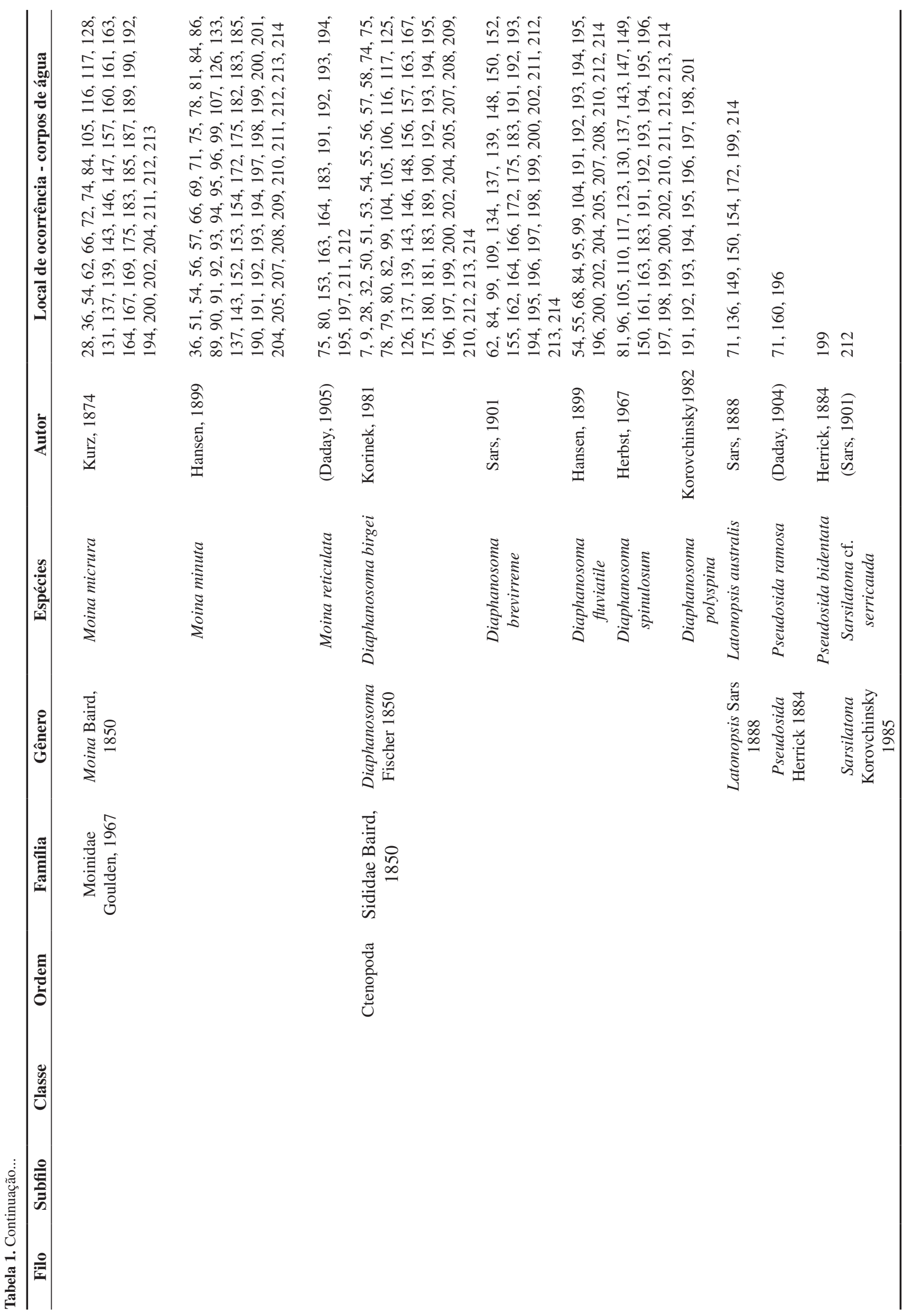


naquele trabalho e a presente síntese. Considerando-se os novos registros ocorridos ao longo da última década (Elmoor-Loureiro 2004, Serafim Jr. et al. 2003, Sinev \& Elmoor-Loureiro 2010) e o registro da invasão por espécies exóticas (Rocha et al. 2003, Zanata et al. 2003) observa-se, no entanto, que o número de espécies válidas aumentou efetivamente.

A lista de espécies de Cladocera de água doce para o Estado de São Paulo inclui atualmente 96 spécies (Tabela 1), sendo 52 espécies da família Chydoridae, e 44 das demais, assim distribuídas: 16 espécies da família Daphnidae, nove espécies de Sididae, oito espécies de Macrothricidae, seis espécies de Bosminidae, três espécies de Moinidae e duas espécies de Ilyocryptidae.

\section{Comentários sobre a lista, riqueza do estado comparado com outras regiões}

A comparação da riqueza total de espécies de Cladocera com outras entre regiões é dificultada pelo fato dos Chydoridae, que respondem por mais de $50 \%$ da riqueza total do grupo terem sido pouco estudados em outras regiões ou estados. Para outras famílias a riqueza é comparável àquela descrita por exemplo para a planície de inundação do Paraná, no Estado do Paraná (Lansac-Toha et al. 1997). A riqueza, no entanto é muito maior do que a reportada em estudos fragmentados oriundos de outros estados do Sul e Sudeste brasileiros. Na realidade não existem ainda inventários consistentes da diversidade da fauna e flora das águas continentais brasileiras, particularmente em relação aos invertebrados.

As unidades (UGRHi) onde se registrou a maior riqueza de espécies foram Mogi-Guaçu e Aguapei, ambas com 27 espécies. De maneira geral as unidades de gerenciamento onde foram registradas as mais baixas riquezas de espécies foram a Litoral Norte, as três unidades compreendidas na bacia do rio Paranapanema (Alto, Médio e Pontal) e a Baixada Santista em cujos corpos de água amostrados não foram encontradas espécies de Cladocera (Figura 1).

Quanto aos padrões de distribuição das espécies observase que espécies pertencentes ao mesmo gênero apresentam padrões bem diferenciados e contrastantes. Como exemplo Simocephalus serrulatus é uma espécie amplamente distribuída, enquanto Simocephalus acutirostris tem distribuição muito restrita. O mesmo se observa para as espécies Ceriodaphnia cornuta e Ceriodaphnia silvestri de ampla distribuição, comparadas com Ceriodaphnia pulchella e Ceriodaphnia richardi, ambas com ocorrência registrada em apenas uma localidade.

Ainda no âmbito do Projeto BIOTA/FAPESP foram realizados estudos aprofundados do ciclo de vida de algumas espécies:

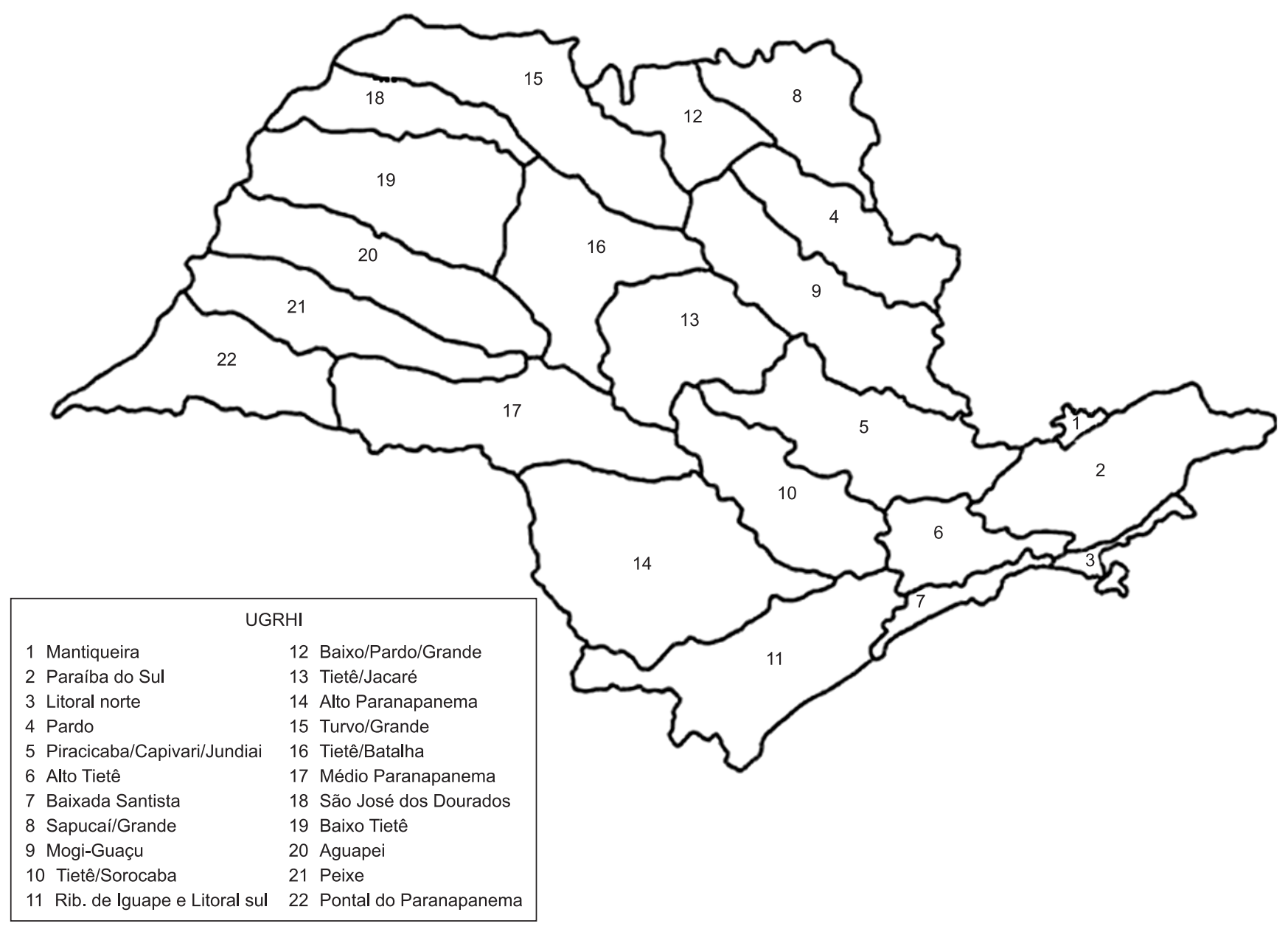

Figura 1. Mapa do Estado de São Paulo com as 22 Unidades de Gerenciamento de Recursos Hidricos, onde foram amostrados em média 10 corpos de água em cada UGRHI, totalizando 220 corpos de água.

Figure 1. Map of São Paulo State showing the 22 Water Resources Management Unities (UGHRi) in which at least 10 water bodies were sampled. 
Diaphanosoma birgei e Ceriodaphnia silvestrii (Rosa 2008); Macrothrix flabelligera (Guntzel et al. 2003); Pseudosida ramosa (Freitas \& Rocha 2006a) e Chydorus pubescens (SantosWisniewski et al. 2006).

\section{Principais avanços relacionados ao Programa BIOTA/FAPESP}

O Programa BIOTA/FAPESP, com enfoque sobre a comunidade zooplanctônica, buscou uma ampla cobertura geográfica visando mapear a distribuição das espécies já conhecidas no Estado de São Paulo, e possibilitar que novas espécies fossem encontradas ou novas ocorrências registradas. Neste sentido foram analisadas amostras oriundas de 172 corpos de água pertencentes a 22 unidades de gerenciamento dos recursos hídricos do Estado de São Paulo. O número de corpos de água amostrados foi maior, contudo em alguns não havia ocorrência de Cladocera.

Com este estudo houve uma ampliação significativa no conhecimento sobre a distribuição geográfica das espécies no Estado de São Paulo (Tabela 1), cujo conhecimento era muito fragmentado. Nesta tabela são registradas 96 espécies, com 12 novos registros de espécies para o Estado de São Paulo, sendo 10 espécies nativas (Pseudosida ramosa, Latonopsis australis, Simocephalus acutirostris, Simocephalus latirostris, Scapholeberis armata, Bosmina freyi, Bosmina huaronensis, Macrothrix superaculeata e Macrothrix flabelligera) e 2 espécies exóticas (Daphnia lumholtzi e Ceriodaphnia dubia). Desta forma o total de 84 espécies de Cladocera para o Estado de São Paulo foi elevado para 96 espécies, com um acréscimo de $15 \%$ na diversidade do grupo.

No total de 22 unidades foram registradas 52 espécies de Chydoridae, 30 da sub-família Aloninae e 17 da sub-família Chydorinae (Tabela 1). As espécies mais amplamente distribuidas foram Alona guttata da sub-família Aloninae e Chydorus eurynotus da sub-família Chydorinae. A distribuição de algumas espécies ficou restrita a apenas uma unidade como Alona setigera e Disparalona leptoryncha.

A segunda maior riqueza foi registrada na família Daphnidae, com 16 espécies. Parte das espécies desta família são cladóceros tipicamente planctônicos (espécies dos gêneros Daphnia e Ceriodaphnia) e também as espécies de maior tamanho, com mais de 3,0 mm de comprimento (Daphnia laevis, Daphnia gessneri e Simocephalus serrulatus). Destacou-se também a família Sididae com nove espécies, algumas de maior tamanho como Latonopsis australis, Pseudosida ramosa e Diaphanosoma birgei.

Ao longo do desenvolvimento do Programa BIOTA/FAPESP relativo ao Zooplâncton foram realizados 14 novos registros de espécies para o Estado de São Paulo, sendo nove espécies nativas (Pseudosida ramosa, Latonopsis australis, Simocephalus acutirostris, Simopcephalus latirostris, Scapholeberis armata, Bosmina freyi, Bosmina huaronensis, Macrothrix superaculeata,Macrothrix flabelligera e Alona setigera) e duas espécies exóticas (Daphnia lumholtzi e Ceriodaphnia dubia). Desta forma o total de 84 espécies de Cladocera para o Estado de São Paulo foi elevado para 96 espécies, com um acréscimo de $15 \%$ na diversidade do grupo.

Diversas pesquisas sobre a biologia das espécies foram realizadas a partir do material vivo trazido ao laboratório no âmbito do Programa BIOTA/FAPESP (Guntzel et al. 2003, Rosa 2003, SantosWisniewski et al. 2006, Freitas \& Rocha 2006a). Estes estudos permitiram ainda que algumas espécies nativas tivessem sua biologia conhecida ou incrementada e por esta razão pudessem ser adotadas como organismos-teste em estudos ecotoxicológicos (Nogueira 2002, Gusso 2004, Choueri 2004, Castilho 2005, Freitas \& Rocha 2006a, b, 2009, 2011, Rocha 2009, Melo 2009), minimizando desta forma o risco de invasão de corpos de água por espécies exóticas, oriundas de escape de organismos-teste exóticos utilizados em experimentos de laboratório.

\section{Principais grupos de pesquisa no Estado de São Paulo}

Não existem pesquisadores dedicados especificamente ao grupo dos Cladocera, no Estado de São Paulo, para se aprofundar em conhecimento taxonômico. A maioria desses estudos são realizados por pesquisadores especializados no estudo da comunidade zooplanctônica sob o ponto de vista ecológico ( relações da ocorrência dos diferentes grupos zooplanctônicos com o meio físico) não conseguindo estudar especificamente um determinado grupo de uma forma detalhada. O Programa BIOTA/FAPESP permitiu cobrir essa lacuna formando grupos de pesquisa para os diferentes grupos taxonômicos do zooplancton.

\section{Principais acervos}

As coleções de espécimes representativos de cada espécie são e serão imprescindíveis para o avanço do conhecimento sobre a diversidade de Cladocera no Estado de São Paulo e no Brasil. Neste sentido o material coletado no âmbito do Programa BIOTA/FAPESP e em outros projetos financiados por diferentes agências encontramse armazenados nas instituições de origem dos pesquisadores, mas não constituem ainda uma coleção de referência propriamente dita. Será ainda necessário organizar uma coleção espécimes preservados em meio líquido e montados em lâminas para todas as espécies com ocorrência no estado.

O Museu de Zoologia da Universidade de São Paulo (MZUSP) possui espécimes de Cladocera depositados por diferentes pesquisadores em diferentes épocas.

Existem coleções do material bruto (amostras do zooplâncton total) oriundas do Programa BIOTA/FAPESP e de outros projetos, depositados no Instituto Internacional de Ecologia e na Universidade Federal de São Carlos, São Carlos, SP. Existem ainda coleções relevantes de material zooplanctônico na Universidade Estadual, campus de Botucatu, e na Universidade de São Paulo, campus de Ribeirão Preto.

\section{Principais lacunas do conhecimento}

Existe ainda uma lacuna no conhecimento taxonômico. Para a maioria das famílias há espécies que são consideradas grupos ou complexos de espécies e que precisam ser objeto de estudos taxonômicos detalhados. Diaphanosoma birgei, de ampla distribuição no estado é considerado um complexo de espécies assim como Latonopsis australis (Korovchinsky 2002, 2005). Daphnia ambigua, Daphnia laevis e Daphnia gessneri são três espécies de ocorrência no Brasil e no Estado de São Paulo (Matsumura-Tundisi 1984). As duas primeiras espécies são de ampla ocorrência na América do Norte, e são consideradas complexos de espécies (Taylor et al. 1998, Herbert et al. 2003). Elías-Gutierrez et al. (2008) obtiveram elevada variabilidade genética no DNA mitocondrial de Moina micrura e sugerem que esta seja também um complexo de espécies. Um outro aspecto é que embora o projeto "Biodiversidade zooplanctônica e o estado de degradação dos ecossistemas aquáticos continentais do Estado de São Paulo" integrante do Programa BIOTA/FAPESP no qual o estudo dos Cladocera estava inserido tenha se destacado em relação a outros projetos do Programa pela sua ampla cobertura geográfica (todas as unidades de recursos hídricos do Estado de São Paulo, e um número de corpos de água avaliados ao redor de 300), teve por outro lado as limitações inerentes a um estudo desta envergadura, como o fato de ter tido apenas uma coleta por corpo de água na maioria das localidades amostradas, e não ter abrangido 
Rocha, O. et al.

Tabela 2. Relação dos corpos de água do estado de São Paulo e respectivas: UGRHi onde ocorrem, códigos numéricos e coordenadas geográficas dos locais amostrados durante a pesquisa realizada no âmbito do Programa BIOTA/FAPESP.Table 2.

Table 2. List of water bodies in São Paulo state and respective: UGHRi where they occur, number codes and geographical coordinates for the sites sampled during the research developed in the scope of the BIOTA/ FAPESP Program.

\begin{tabular}{|c|c|c|c|c|c|}
\hline UGRHi & $\begin{array}{c}\text { Código dos } \\
\text { corpos d'água }\end{array}$ & Corpos d'água & Latitude & Longitude & Projeto ou autor \\
\hline \multirow[t]{19}{*}{1 - Mantiqueira } & 1 & Represa do Fojo & $22^{\circ} 42^{\prime} 54^{\prime \prime} \mathrm{S}$ & $45^{\circ} 32^{\prime} 06^{\prime \prime} \mathrm{W}$ & BIOTA FAPESP \\
\hline & 2 & Represa Marginal Fojo & $22^{\circ} 42^{\prime} 57^{\prime \prime} \mathrm{S}$ & $45^{\circ} 32^{\prime} 05^{\prime \prime} \mathrm{W}$ & \\
\hline & 3 & Lago dos Lambaris & $22^{\circ} 41^{\prime} 23^{\prime \prime} \mathrm{S}$ & $45^{\circ} 28^{\prime} 58^{\prime \prime} \mathrm{W}$ & \\
\hline & 4 & Lago das C201Ninféas & $22^{\circ} 41^{\prime} 27^{\prime \prime} \mathrm{S}$ & $45^{\circ} 29^{\prime} 08^{\prime \prime} \mathrm{W}$ & \\
\hline & 5 & Represa Horto Florestal 1 & & & \\
\hline & 6 & Represa Horto Florestal 2 & & & \\
\hline & 7 & Represa Horto Florestal 3 & & & \\
\hline & 8 & Represa Horto Florestal 4 & & & \\
\hline & 9 & Represa Horto Florestal 5 & & & \\
\hline & 10 & Represa Santa Isabel & $22^{\circ} 43^{\prime} 35^{\prime} \mathrm{S}$ & $45^{\circ} 27^{\prime} 01^{\prime \prime} \mathrm{W}$ & \\
\hline & 11 & Riacho das Trutas & $22^{\circ} 43^{\prime} 20^{\prime \prime} \mathrm{S}$ & $45^{\circ} 27^{\prime} 06^{\prime \prime} \mathrm{W}$ & \\
\hline & 12 & Lago Tundra & $22^{\circ} 43^{\prime} 18^{\prime \prime} \mathrm{S}$ & $45^{\circ} 27^{\prime} 08^{\prime \prime} \mathrm{W}$ & \\
\hline & 13 & Lago Lavrinhas 1 & $22^{\circ} 42^{\prime} 08^{\prime \prime} \mathrm{S}$ & $45^{\circ} 25^{\prime} 12^{\prime \prime} \mathrm{W}$ & \\
\hline & 14 & Lago Lavrinhas 2 & $22^{\circ} 41^{\prime} 51^{\prime \prime S}$ & $45^{\circ} 25^{\prime} 09^{\prime} \mathrm{W}$ & \\
\hline & 15 & Represa do Instituto de Pesca & & & \\
\hline & 16 & Represa do Pico Itapeva & $22^{\circ} 46^{\prime} 11^{\prime \prime S}$ & $45^{\circ} 31^{\prime} 48^{\prime \prime} \mathrm{W}$ & \\
\hline & 17 & Lago Hípica 1 & $22^{\circ} 43^{\prime} 24^{\prime \prime} \mathrm{S}$ & $45^{\circ} 33^{\prime} 06^{\prime \prime} \mathrm{W}$ & \\
\hline & 18 & Lago Hípica 2 & $22^{\circ} 43 ’ 21^{\prime \prime} \mathrm{S}$ & $45^{\circ} 33^{\prime} 04^{\prime \prime} \mathrm{W}$ & \\
\hline & 19 & Lago Vila Inglesa & $22^{\circ} 44^{\prime} 28^{\prime \prime} \mathrm{S}$ & $45^{\circ} 34^{\prime} 06^{\prime \prime} \mathrm{W}$ & \\
\hline \multirow[t]{8}{*}{2 - Paraíba do Sul } & 20 & Represa Santa Branca & $23^{\circ} 22^{\prime} 06^{\prime \prime} \mathrm{S}$ & $45^{\circ} 51^{\prime} 28^{\prime \prime} \mathrm{W}$ & BIOTA FAPESP \\
\hline & 21 & Represa Paraibuna & $23^{\circ} 26^{\prime} 53^{\prime \prime} \mathrm{S}$ & $45^{\circ} 33^{\prime} 44^{\prime \prime} \mathrm{W}$ & \\
\hline & 22 & Lagoa Olaria & $22^{\circ} 49^{\prime} 06^{\prime \prime} \mathrm{S}$ & $45^{\circ} 13^{\prime} 24^{\prime \prime} \mathrm{W}$ & \\
\hline & 23 & Lagoa Marginal & $22^{\circ} 49^{\prime} 19^{\prime \prime} \mathrm{S}$ & $45^{\circ} 12^{\prime} 45^{\prime \prime} \mathrm{W}$ & \\
\hline & 24 & Represa do Funil & $22^{\circ} 30^{\prime} 43^{\prime \prime} \mathrm{S}$ & $44^{\circ} 38^{\prime} 01^{\prime \prime} \mathrm{W}$ & \\
\hline & 25 & Represa Hotel & $22^{\circ} 37^{\prime} 33^{\prime \prime} \mathrm{S}$ & $44^{\circ} 38^{\prime} 48^{\prime \prime} \mathrm{W}$ & \\
\hline & 26 & $\begin{array}{c}\text { Lagoa Parque Nacional } \\
\text { Bocaina }\end{array}$ & $22^{\circ} 44^{\prime} 33^{\prime \prime} \mathrm{S}$ & $44^{\circ} 36^{\prime} 58^{\prime \prime} \mathrm{W}$ & \\
\hline & 27 & $\begin{array}{c}\text { Lagoa Chácara Serra da } \\
\text { Bocaina }\end{array}$ & $22^{\circ} 38^{\prime} 54^{\prime \prime} \mathrm{S}$ & $44^{\circ} 35^{\prime} 38^{\prime \prime} \mathrm{W}$ & \\
\hline \multirow[t]{5}{*}{3 - Litoral Norte } & 28 & Represa Rio Macaco & $23^{\circ} 25^{\prime} 30^{\prime \prime} \mathrm{S}$ & $45^{\circ} 08^{\prime} 10^{\prime \prime} \mathrm{W}$ & BIOTA FAPESP \\
\hline & 29 & Barragem do Rio Grande & $23^{\circ} 23^{\prime} 43^{\prime \prime} \mathrm{S}$ & $45^{\circ} 07^{\prime} 10^{\prime \prime} \mathrm{W}$ & \\
\hline & 30 & Barragem do Rio Piaba & $23^{\circ} 31^{\prime} 45^{\prime \prime} \mathrm{S}$ & $45^{\circ} 15^{\prime} 28^{\prime \prime} \mathrm{W}$ & \\
\hline & 31 & Represa Cantinho do Céu & $23^{\circ} 31^{\prime} 54^{\prime \prime} \mathrm{S}$ & $45^{\circ} 15^{\prime} 32^{\prime \prime} \mathrm{W}$ & \\
\hline & 32 & Represa Água Branca & $23^{\circ} 50^{\prime} 12^{\prime \prime} \mathrm{S}$ & $45^{\circ} 21^{\prime} 30^{\prime \prime} \mathrm{W}$ & \\
\hline \multirow[t]{6}{*}{4 - Pardo } & 33 & Represa Graminha ou Caconde & $21^{\circ} 34^{\prime} 48^{\prime \prime} \mathrm{S}$ & $47^{\circ} 37^{\prime} 10^{\prime \prime \prime} \mathrm{W}$ & BIOTA FAPESP \\
\hline & 34 & Represa Itaiquara & $21^{\circ} 35^{\prime} 05^{\prime \prime} \mathrm{S}$ & $46^{\circ} 44^{\prime} 52^{\prime \prime} \mathrm{W}$ & \\
\hline & 35 & Fazenda Graminha & $21^{\circ} 32^{\prime} 55^{\prime \prime} \mathrm{S}$ & $46^{\circ} 49^{\prime} 36^{\prime \prime} \mathrm{W}$ & \\
\hline & 36 & Represa Euclides da Cunha & $21^{\circ} 36^{\prime} 03^{\prime \prime} \mathrm{S}$ & $46^{\circ} 56^{\prime} 55^{\prime \prime} \mathrm{W}$ & \\
\hline & 37 & Fazenda Santa Helena & $21^{\circ} 32^{\prime} 04^{\prime \prime} \mathrm{S}$ & $46^{\circ} 50^{\prime} 30^{\prime \prime} \mathrm{W}$ & \\
\hline & 38 & Represa Limoeiro & & & \\
\hline \multirow{9}{*}{$\begin{array}{l}5 \text { - Piracicaba/ } \\
\text { Jundiaí/Capivar }\end{array}$} & 39 & Represa Igaratá (R. Jaguari) & $23^{\circ} 11^{\prime} 25^{\prime \prime} \mathrm{S}$ & $46^{\circ} 07^{\prime} 15^{\prime \prime} \mathrm{W}$ & BIOTA FAPESP \\
\hline & 40 & Represa Cachoeira & $23^{\circ} 07^{\prime} 31^{\prime \prime} \mathrm{S}$ & $46^{\circ} 17^{\prime} 19^{\prime \prime} \mathrm{W}$ & \\
\hline & 41 & Represa de Piracaia & $23^{\circ} 04^{\prime} 14^{\prime \prime} \mathrm{S}$ & $46^{\circ} 19^{\prime} 19^{\prime \prime} \mathrm{W}$ & \\
\hline & 42 & Represa Atibainha & $23^{\circ} 10^{\prime} 33^{\prime \prime} \mathrm{S}$ & $46^{\circ} 23$ ' 13 '” W & \\
\hline & 43 & Represa Atibaia & $23^{\circ} 05^{\prime} 29^{\prime \prime} \mathrm{S}$ & $46^{\circ} 38^{\prime} 08^{\prime \prime} \mathrm{W}$ & \\
\hline & 44 & Represa Salto Grande & & & \\
\hline & 45 & Represa Paramirim & & & \\
\hline & 46 & $\begin{array}{c}\text { Represa Limoeiro - Águas de } \\
\text { São Pedro }\end{array}$ & & & \\
\hline & 47 & Lago da Pousada Casa Amarela & & & \\
\hline
\end{tabular}


Tabela 2. Continuação...

\begin{tabular}{|c|c|c|c|c|c|}
\hline UGRHi & $\begin{array}{l}\text { Código dos } \\
\text { corpos d'água }\end{array}$ & Corpos d'água & Latitude & Longitude & Projeto ou autor \\
\hline \multirow[t]{13}{*}{6 - Alto Tietê } & 48 & Lago 1 Parque Ecológico & $23^{\circ} 29^{\prime} 12^{\prime \prime} \mathrm{S}$ & $46^{\circ} 30^{\prime} 48^{\prime \prime} \mathrm{W}$ & BIOTA FAPESP \\
\hline & 49 & Lago 2 Parque Ecológico & $23^{\circ} 29^{\prime} 04^{\prime \prime} \mathrm{S}$ & $46^{\circ} 31^{\prime} 05^{\prime \prime} \mathrm{W}$ & \\
\hline & 50 & Represa Ribeirão do Campo & $23^{\circ} 38^{\prime} 46^{\prime \prime} \mathrm{S}$ & $45^{\circ} 49^{\prime} 53^{\prime \prime} \mathrm{W}$ & \\
\hline & 51 & Represa. Billings & $23^{\circ} 46^{\prime} 49^{\prime \prime} \mathrm{S}$ & $46^{\circ} 32^{\prime} 37^{\prime \prime} \mathrm{W}$ & \\
\hline & 52 & Represa Cachoeira das Graças & $23^{\circ} 39^{\prime} 13^{\prime \prime} \mathrm{S}$ & $46^{\circ} 58^{\prime} 03^{\prime \prime} \mathrm{W}$ & \\
\hline & 53 & Represa Pedro Brecht & $23^{\circ} 43^{\prime} 03{ }^{\prime \prime} \mathrm{S}$ & $46^{\circ} 57^{\prime} 38^{\prime \prime} \mathrm{W}$ & \\
\hline & 54 & Represa Paulo Paiva Castro & $23^{\circ} 19^{\prime} 56^{\prime \prime} \mathrm{S}$ & $46^{\circ} 39^{\prime} 15^{\prime \prime} \mathrm{W}$ & \\
\hline & 55 & Represa Taiaçupeba & $23^{\circ} 34^{\prime} 48^{\prime \prime} \mathrm{S}$ & $46^{\circ} 16^{\prime} 55^{\prime \prime} \mathrm{W}$ & \\
\hline & 56 & Represa Ponte Nova & $23^{\circ} 35^{\prime} 50^{\prime \prime} \mathrm{S}$ & $45^{\circ} 56^{\prime} 47^{\prime \prime} \mathrm{W}$ & \\
\hline & 57 & Lagoa Ribeirão Pires & $23^{\circ} 41^{\prime} 19^{\prime \prime} \mathrm{S}$ & $46^{\circ} 22^{\prime} 25^{\prime \prime} \mathrm{W}$ & \\
\hline & 58 & Represa Águas Claras & $23^{\circ} 23^{\prime} 54^{\prime \prime} \mathrm{S}$ & $46^{\circ} 39^{\prime} 31^{\prime \prime} \mathrm{W}$ & \\
\hline & 59 & Represa Jundiaí & $23^{\circ} 39^{\prime} 01^{\prime \prime} \mathrm{S}$ & $46^{\circ} 11^{\prime} 31^{\prime \prime} \mathrm{W}$ & \\
\hline & 60 & Lago rio Claro & $23^{\circ} 38^{\prime} 45^{\prime \prime} \mathrm{S}$ & $45^{\circ} 51^{\prime} 39^{\prime \prime} \mathrm{W}$ & \\
\hline \multirow[t]{4}{*}{8 - Sapucaí/Grande } & 61 & Represa Jaguara & $20^{\circ} 04^{\prime} 45^{\prime \prime} \mathrm{S}$ & $47^{\circ} 24^{\prime} 37^{\prime \prime} \mathrm{W}$ & BIOTA FAPESP \\
\hline & 62 & Represa Estreito & $20^{\circ} 09^{\prime} 26^{\prime \prime} \mathrm{S}$ & $47^{\circ} 15^{\prime} 56^{\prime \prime} \mathrm{W}$ & \\
\hline & 63 & Represa Igarapava & $20^{\circ} 59^{\prime} 17^{\prime \prime} \mathrm{S}$ & $47^{\circ} 43^{\prime} 56^{\prime \prime} \mathrm{W}$ & \\
\hline & 64 & Represa Volta Grande & $20^{\circ} 08^{\prime} 40^{\prime \prime} \mathrm{S}$ & $48^{\circ} 02^{\prime} 21^{\prime \prime} \mathrm{W}$ & \\
\hline \multirow[t]{17}{*}{9 - Mogi-Guaçu } & 65 & Represa do David & $22^{\circ} 19^{\prime} 26^{\prime \prime} \mathrm{S}$ & $46^{\circ} 45^{\prime} 27^{\prime \prime} \mathrm{W}$ & BIOTA FAPESP \\
\hline & 66 & Represa da Fazenda Aurora & $20^{\circ} 59^{\prime} 49^{\prime \prime} \mathrm{S}$ & $47^{\circ} 58^{\prime} 57^{\prime \prime} \mathrm{W}$ & \\
\hline & 67 & Lagoa do Diogo & $21^{\circ} 37^{\prime} 27^{\prime \prime} \mathrm{S}$ & $47^{\circ} 48^{\prime} 24^{\prime \prime} \mathrm{W}$ & \\
\hline & 68 & Represa Cristal & $21^{\circ} 36^{\prime} 25^{\prime \prime} \mathrm{S}$ & $47^{\circ} 47^{\prime} 57^{\prime \prime} \mathrm{W}$ & \\
\hline & 69 & Represa Santa Margarida & $21^{\circ} 27^{\prime} 33^{\prime \prime} \mathrm{S}$ & $48^{\circ} 02^{\prime} 01^{\prime \prime} \mathrm{W}$ & \\
\hline & 70 & Lagoa do Barro Preto & $21^{\circ} 29^{\prime} 38^{\prime \prime} \mathrm{S}$ & $48^{\circ} 01^{\prime} 59^{\prime \prime} \mathrm{W}$ & \\
\hline & 71 & Lagoa das Cabras & $21^{\circ} 29^{\prime} 09^{\prime \prime} \mathrm{S}$ & $48^{\circ} 03^{\prime} 43^{\prime \prime} \mathrm{W}$ & \\
\hline & 72 & Lagoa do Peixe & $21^{\circ} 37^{\prime} 25^{\prime \prime} \mathrm{S}$ & $47^{\circ} 48^{\prime} 24^{\prime \prime} \mathrm{W}$ & \\
\hline & 73 & Lagoa Verde & $21^{\circ} 20^{\prime} 37,4^{\prime \prime} \mathrm{S}$ & $48^{\circ} 07^{\prime} 7,5^{\prime \prime} \mathrm{W}$ & \\
\hline & 74 & Lago do Paço Municipal & $23^{\circ} 05^{\prime} 01^{\prime \prime} \mathrm{S}$ & $48^{\circ} 33^{\prime} 31^{\prime \prime} \mathrm{W}$ & \\
\hline & 75 & Repr. São Geraldo & $22^{\circ} 19^{\prime} 26^{\prime \prime} \mathrm{S}$ & $46^{\circ} 45^{\prime} 26^{\prime \prime} \mathrm{W}$ & \\
\hline & 76 & Represa da Prainha & $19^{\circ} 59^{\prime} 30^{\prime \prime} \mathrm{S}$ & $49^{\circ} 23^{\prime} 54^{\prime \prime} \mathrm{W}$ & \\
\hline & 77 & Represa Aguaí & $22^{\circ} 0{ }^{\prime} 47^{\prime \prime} \mathrm{S}$ & $47^{\circ} 10^{\prime} 5,8^{\prime \prime} \mathrm{W}$ & \\
\hline & 78 & Lago da Churrascaria & $21^{\circ} 57^{\prime} 17^{\prime \prime} \mathrm{S}$ & $46^{\circ} 43^{\prime} 13^{\prime \prime} \mathrm{W}$ & \\
\hline & 79 & Represa Irmãos Ribeiro & $22^{\circ} 10^{\prime} 01^{\prime \prime} \mathrm{S}$ & $46^{\circ} 46^{\prime} 43^{\prime \prime} \mathrm{W}$ & \\
\hline & 80 & $\begin{array}{c}\text { Represa Churrascaria Sto } \\
\text { Antonio }\end{array}$ & $22^{\circ} 33^{\prime} 52^{\prime \prime} \mathrm{S}$ & $46^{\circ} 32^{\prime} 18^{\prime \prime} \mathrm{W}$ & \\
\hline & 81 & Represa Mogi-Guaçu & $22^{\circ} 22^{\prime} 56^{\prime \prime} \mathrm{S}$ & $46^{\circ} 53^{\prime} 56^{\prime \prime} \mathrm{W}$ & \\
\hline \multirow{3}{*}{$\begin{array}{l}10 \text { - Sorocaba/Médio } \\
\text { Tietê }\end{array}$} & 82 & Represa Itupararanga & $23^{\circ} 35^{\prime} 26^{\prime \prime} \mathrm{S}$ & $47^{\circ} 17^{\prime} 10^{\prime \prime \prime} \mathrm{W}$ & BIOTA FAPESP \\
\hline & 83 & Represa Prainha & $23^{\circ} 34^{\prime} 54^{\prime} \mathrm{S}$ & $47^{\circ} 26^{\prime} 03^{\prime \prime} \mathrm{W}$ & \\
\hline & 84 & Represa Hedberg & $23^{\circ} 25^{\prime} 36^{\prime \prime} \mathrm{S}$ & $47^{\circ} 35^{\prime} 41^{\prime \prime} \mathrm{W}$ & \\
\hline \multirow{10}{*}{$\begin{array}{l}\text { 11- Ribeira de } \\
\text { Iguape/Litoral Sul }\end{array}$} & 85 & Ribeira do Iguape & $24^{\circ} 35^{\prime} 25^{\prime \prime} \mathrm{S}$ & $48^{\circ} 35^{\prime} 45^{\prime \prime} \mathrm{W}$ & BIOTA FAPESP \\
\hline & 86 & $\begin{array}{l}\text { Lago da Congregação Cristã } \\
\text { do Brasil }\end{array}$ & $24^{\circ} 33^{\prime} 02^{\prime \prime} \mathrm{S}$ & $48^{\circ} 08^{\prime} 04^{\prime \prime} \mathrm{W}$ & \\
\hline & 87 & Represa Juquiazinho & $23^{\circ} 56^{\prime} 01^{\prime \prime} \mathrm{S}$ & $47^{\circ} 30^{\prime} 25^{\prime \prime \prime} \mathrm{W}$ & \\
\hline & 88 & Represa Japaris & $23^{\circ} 56^{\prime} 49^{\prime \prime} \mathrm{S}$ & $47^{\circ} 30^{\prime} 09^{\prime \prime} \mathrm{W}$ & \\
\hline & 89 & Represa do Porto Rio J. Guaçu & $24^{\circ} 03^{\prime} 31^{\prime \prime} \mathrm{S}$ & $47^{\circ} 24^{\prime} 35^{\prime \prime} \mathrm{W}$ & \\
\hline & 90 & Represa Serraria & $24^{\circ} 08^{\prime} 19^{\prime \prime} \mathrm{S}$ & $47^{\circ} 32^{\prime} 35^{\prime \prime} \mathrm{W}$ & \\
\hline & 91 & $\begin{array}{c}\text { Represa Alecrim-Barragem/ } \\
\text { Rio J G. }\end{array}$ & $24^{\circ} 04^{\prime} 46^{\prime \prime} \mathrm{S}$ & $47^{\circ} 28^{\prime} 34^{\prime \prime} \mathrm{W}$ & \\
\hline & 92 & Represa Cachoeira da França & $23^{\circ} 56^{\prime} 04^{\prime \prime} \mathrm{S}$ & $47^{\circ} 11^{\prime} 21^{\prime \prime} \mathrm{W}$ & \\
\hline & 93 & Represa FUMAÇA & $24^{\circ} 00^{\prime} 16^{\prime \prime} \mathrm{S}$ & $47^{\circ} 15^{\prime} 41^{\prime \prime} \mathrm{W}$ & \\
\hline & 94 & Represa Barra & $24^{\circ} 00^{\prime} 01^{\prime \prime} \mathrm{S}$ & $47^{\circ} 20^{\prime} 37^{\prime \prime} \mathrm{W}$ & \\
\hline
\end{tabular}


Rocha, O. et al.

Tabela 2. Continuação...

\begin{tabular}{|c|c|c|c|c|c|}
\hline UGRHi & $\begin{array}{c}\text { Código dos } \\
\text { corpos d'água }\end{array}$ & Corpos d'água & Latitude & Longitude & Projeto ou autor \\
\hline \multirow{5}{*}{$\begin{array}{l}12 \text { - Baixo Pardo/ } \\
\text { Grande }\end{array}$} & 95 & Lago Urbano Municipal & $20^{\circ} 33^{\prime} 53^{\prime \prime}$ & $48^{\circ} 34^{\prime} 46^{\prime \prime} \mathrm{W}$ & BIOTA FAPESP \\
\hline & 96 & $\begin{array}{c}\text { Reservatório de Porto } \\
\text { Colômbia }\end{array}$ & $20^{\circ} 07^{\prime} 36^{\prime \prime}$ & $48^{\circ} 33^{\prime} 43^{\prime \prime} \mathrm{W}$ & \\
\hline & 97 & Foz do Rio Pardo & $20^{\circ} 10^{\prime} 23^{\prime \prime}$ & $48^{\circ} 37^{\prime} 41^{\prime \prime} \mathrm{W}$ & \\
\hline & 98 & Represa dos Soppa & $20^{\circ} 11^{\prime} 12^{\prime \prime}$ & $48^{\circ} 38^{\prime} 60^{\prime \prime} \mathrm{W}$ & \\
\hline & 99 & Reservatório de Marimbondo & $20^{\circ} 18^{\prime} 30^{\prime \prime}$ & $49^{\circ} 10^{\prime} 29$ '” & \\
\hline \multirow[t]{18}{*}{13 - Tietê/Jacaré } & 100 & Represa do Jardim Botânico & $22^{\circ} 20^{\prime} 18^{\prime \prime} \mathrm{S}$ & $49^{\circ} 00^{\prime} 52^{\prime \prime} \mathrm{W}$ & BIOTA FAPESP \\
\hline & 101 & Represa do Zoológico & $22^{\circ} 20^{\prime} 31^{\prime \prime} \mathrm{S}$ & $49^{\circ} 01^{\prime} 04$ "'W & \\
\hline & 102 & $\begin{array}{c}\text { Represa da Secretaria da } \\
\text { Agricultura }\end{array}$ & $22^{\circ} 21^{\prime} 20^{\prime \prime} \mathrm{S}$ & $48^{\circ} 45^{\prime} 23^{\prime \prime} \mathrm{W}$ & \\
\hline & 103 & Lago da Fazenda Primavera & $22^{\circ} 07^{\prime} 43^{\prime \prime} \mathrm{S}$ & $47^{\circ} 51^{\prime} 38^{\prime \prime} \mathrm{W}$ & \\
\hline & 104 & Represa do Instituto Florestal & $22^{\circ} 14^{\prime} 52^{\prime \prime} \mathrm{S}$ & $47^{\circ} 49^{\prime} 25^{\prime \prime} \mathrm{W}$ & \\
\hline & 105 & Lago da Areia que Canta & $22^{\circ} 18^{\prime} 54^{\prime \prime} \mathrm{S}$ & 4803’04'W & \\
\hline & 106 & Lago Sítio Mariano Lopes & $22^{\circ} 23^{\prime} 19^{\prime \prime} \mathrm{S}$ & $48^{\circ} 22^{\prime} 52^{\prime \prime} \mathrm{W}$ & \\
\hline & 107 & $\begin{array}{l}\text { Represa Marisa } \\
\text { (Usina Tamoio) }\end{array}$ & $21^{\circ} 55^{\prime} 31^{\prime \prime} \mathrm{S}$ & $48^{\circ} 06^{\prime} 35^{\prime \prime} \mathrm{W}$ & \\
\hline & 108 & $\begin{array}{c}\text { Fazenda Méia - Ribeirão } \\
\text { Itaquerê }\end{array}$ & $21^{\circ} 47^{\prime} 399^{\prime \prime} \mathrm{S}$ & $48^{\circ} 33^{\prime} 54^{\prime \prime} \mathrm{W}$ & \\
\hline & 109 & Fazenda Palmeiras & $21^{\circ} 45^{\prime} 43^{\prime \prime} \mathrm{S}$ & $48^{\circ} 41^{\prime} 57^{\prime \prime} \mathrm{W}$ & \\
\hline & 110 & Sítio Boa Vista & $21^{\circ} 47^{\prime} 17^{\prime \prime S}$ & $48^{\circ} 47^{\prime} 50^{\prime \prime} \mathrm{W}$ & \\
\hline & 111 & Represa do Lobo - Broa & $22^{\circ} 10^{\prime} 13^{\prime \prime} \mathrm{S}$ & $47^{\circ} 54^{\prime} 15^{\prime \prime} \mathrm{W}$ & \\
\hline & 112 & Represa 29 & $21^{\circ} 53^{\prime} 45^{\prime \prime} \mathrm{S}$ & $47^{\circ} 49^{\prime} 02^{\prime \prime} \mathrm{W}$ & \\
\hline & 113 & Represa do Chile & $21^{\circ} 52^{\prime} 01^{\prime \prime} \mathrm{S}$ & $47^{\circ} 51^{\prime} 55^{\prime \prime} \mathrm{W}$ & \\
\hline & 114 & Represa do Clube Naútico & $21^{\circ} 42^{\prime} 24^{\prime \prime} \mathrm{S}$ & $48^{\circ} 01^{\prime} 37^{\prime \prime} \mathrm{W}$ & \\
\hline & 115 & Represa Ibitinga & $21^{\circ} 45^{\prime} 25^{\prime \prime} \mathrm{S}$ & $48^{\circ} 58^{\prime} 28^{\prime \prime} \mathrm{W}$ & \\
\hline & 116 & Represa de Bariri & $22^{\circ} 09^{\prime} 44^{\prime \prime S}$ & $48^{\circ} 44^{\prime} 34^{\prime \prime} \mathrm{W}$ & \\
\hline & 117 & Represa de Barra Bonita & $22^{\circ} 31$ '49”S & $48^{\circ} 31^{\prime} 14^{\prime \prime \prime} \mathrm{W}$ & \\
\hline \multirow{7}{*}{$\begin{array}{l}14,17 \text { e } 22 \text { - Alto } \\
\text { Paranapanema/ } \\
\text { Médio } \\
\text { Paranapanema/Pontal } \\
\text { do Paranapanema }\end{array}$} & 118 & Represa Jurumirim & $23^{\circ} 26^{\prime} 33^{\prime \prime} \mathrm{S}$ & $48^{\circ} 39^{\prime} 49^{\prime \prime} \mathrm{W}$ & BIOTA FAPESP \\
\hline & 119 & Barragem de Chavantes & $23^{\circ} 08^{\prime} 27^{\prime \prime} \mathrm{S}$ & $49^{\circ} 42^{\prime} 24^{\prime \prime} \mathrm{W}$ & \\
\hline & 120 & Barragem Canoas I & $22^{\circ} 56^{\prime} 31^{\prime \prime} \mathrm{S}$ & $50^{\circ} 30^{\prime} 41^{\prime \prime} \mathrm{W}$ & \\
\hline & 121 & Represa Salto Grande & $22^{\circ} 56^{\prime} 43^{\prime \prime} \mathrm{S}$ & $49^{\circ} 57^{\prime} 60^{\prime \prime \prime} \mathrm{W}$ & \\
\hline & 122 & Represa de Capivara & $22^{\circ} 54^{\prime} 01^{\prime \prime} \mathrm{S}$ & $50^{\circ} 47^{\prime} 30^{\prime \prime} \mathrm{W}$ & \\
\hline & 123 & Represa Taquaruçu & $22^{\circ} 32^{\prime} 36^{\prime \prime} \mathrm{S}$ & $51^{\circ} 59^{\prime} 19^{\prime \prime} \mathrm{W}$ & \\
\hline & 124 & Represa de Rosana & $22^{\circ} 35^{\prime} 40^{\prime \prime} \mathrm{S}$ & $52^{\circ} 51^{\prime} 14^{\prime \prime} \mathrm{W}$ & \\
\hline \multirow[t]{15}{*}{15 - Turvo Grande } & 125 & Lago Empresa Cestari 1 & $21^{\circ} 15^{\prime} 57^{\prime \prime} \mathrm{S}$ & $48^{\circ} 31^{\prime} 29^{\prime \prime} \mathrm{W}$ & BIOTA FAPESP \\
\hline & 126 & Lago Empresa Cestari 2 & $21^{\circ} 16^{\prime} 12^{\prime \prime} \mathrm{S}$ & $48^{\circ} 31^{\prime} 42^{\prime \prime} \mathrm{W}$ & \\
\hline & 127 & Lago Fazenda Socorro & $20^{\circ} 57^{\prime} 499^{\prime} \mathrm{S}$ & $48^{\circ} 40^{\prime} 45^{\prime \prime} \mathrm{W}$ & \\
\hline & 128 & Lago Sítio Liberdade & $20^{\circ} 52 ’ 34 ” S$ & $48^{\circ} 40^{\prime} 31^{\prime \prime} \mathrm{W}$ & \\
\hline & 129 & Lago Tabapuã & $20^{\circ} 56^{\prime} 02^{\prime} \mathrm{S}$ & $49^{\circ} 05^{\prime} 16^{\prime \prime} \mathrm{W}$ & \\
\hline & 130 & Lago Usina São Domingos & $21^{\circ} 05^{\prime} 38^{\prime \prime} \mathrm{S}$ & $49^{\circ} 01^{\prime} 20^{\prime \prime} \mathrm{W}$ & \\
\hline & 131 & $\begin{array}{l}\text { Lago Santa Ana e } \\
\text { Santo Antonio }\end{array}$ & $20^{\circ} 56^{\prime} 25^{\prime} \mathrm{S}$ & $49^{\circ} 15^{\prime} 29^{\prime \prime} \mathrm{W}$ & \\
\hline & 132 & Lago Fazenda Brasil & $20^{\circ} 45^{\prime} 46^{\prime \prime} \mathrm{S}$ & $49^{\circ} 32^{\prime} 58^{\prime \prime} \mathrm{W}$ & \\
\hline & 133 & Lago Chácara Domarco & $20^{\circ} 48^{\prime} 51$ 'S & $49^{\circ} 28^{\prime} 55^{\prime \prime} \mathrm{W}$ & \\
\hline & 134 & $\begin{array}{l}\text { Represa do Rio Preto } \\
\text { Automóvel Clube }\end{array}$ & $20^{\circ} 51^{\prime} 37^{\prime \prime} \mathrm{S}$ & $49^{\circ} 20^{\prime} 08^{\prime \prime} \mathrm{W}$ & \\
\hline & 135 & Represa Tanabi & $20^{\circ} 32{ }^{\prime} 41^{\prime \prime} \mathrm{S}$ & $49^{\circ} 37^{\prime} 40^{\prime \prime} \mathrm{W}$ & \\
\hline & 136 & Represa Ibiporanga & $20^{\circ} 24^{\prime} 60^{\prime \prime} \mathrm{S}$ & $49^{\circ} 29^{\prime} 10^{\prime \prime} \mathrm{W}$ & \\
\hline & 137 & Reservatório de Água Vermelha & $19^{\circ} 56^{\prime} 57^{\prime \prime} \mathrm{S}$ & $49^{\circ} 40^{\prime} 30^{\prime \prime} \mathrm{W}$ & \\
\hline & 138 & Lago Urânia & $20^{\circ} 14^{\prime} 28^{\prime} \mathrm{S}$ & $50^{\circ} 36^{\prime} 58^{\prime \prime} \mathrm{W}$ & \\
\hline & 139 & Rio Grande & $20^{\circ} 04^{\prime} 27^{\prime \prime} \mathrm{S}$ & $50^{\circ} 58^{\prime} 60^{\prime \prime} \mathrm{W}$ & \\
\hline
\end{tabular}


Tabela 2. Continuação...

\begin{tabular}{|c|c|c|c|c|c|}
\hline UGRHi & $\begin{array}{l}\text { Código dos } \\
\text { corpos d'água }\end{array}$ & Corpos d'água & Latitude & Longitude & Projeto ou autor \\
\hline \multirow[t]{3}{*}{16 - Tietê/Batalha } & 140 & Represa de Ibitinga & $21^{\circ} 45^{\prime} 22^{\prime \prime} \mathrm{S}$ & $49^{\circ} 47^{\prime} 40^{\prime \prime} \mathrm{W}$ & BIOTA FAPESP \\
\hline & 141 & $\begin{array}{l}\text { Represa da Fazenda } \\
\text { Santa Catarina }\end{array}$ & & & \\
\hline & 142 & Represa de Promissão & $21^{\circ} 118^{\prime} 32^{\prime \prime} \mathrm{S}$ & $49^{\circ} 47^{\prime} 06^{\prime \prime} \mathrm{W}$ & \\
\hline \multirow{5}{*}{$\begin{array}{l}18 \text { - São José dos } \\
\text { Dourados }\end{array}$} & 143 & Reservatório de Ilha Solteira & $20^{\circ} 26^{\prime} 36^{\prime} \mathrm{S}$ & $51^{\circ} 15^{\prime} 29^{\prime \prime} \mathrm{W}$ & BIOTA FAPESP \\
\hline & 144 & Lago Estância Semax & $20^{\circ} 33^{\prime} 12{ }^{\prime \prime} \mathrm{S}$ & $50^{\circ} 01^{\prime} 38^{\prime \prime} \mathrm{W}$ & \\
\hline & 145 & Lago São Luiz de Japiuba & $20^{\circ} 39^{\prime} 04^{\prime} \mathrm{S}$ & $50^{\circ} 27^{\prime} 11^{\prime \prime} \mathrm{W}$ & \\
\hline & 146 & Lago Sítio São Pedro & $20^{\circ} 42^{\prime} 40^{\prime} \mathrm{S}$ & 4952'06”'W & \\
\hline & 147 & Lago Usina Monte Aprazível & $20^{\circ} 45^{\prime} 18^{\prime \prime} \mathrm{S}$ & $49^{\circ} 43^{\prime} 20^{\prime \prime} \mathrm{W}$ & \\
\hline \multirow[t]{8}{*}{19 - Baixo Tietê } & 148 & Represa de Nova Avanhandava & $21^{\circ} 17^{\prime} 42 ” S$ & $50^{\circ} 08^{\prime} 17^{\prime \prime} \mathrm{W}$ & BIOTA FAPESP \\
\hline & 149 & Lago - Birigui/Araçatuba & $21^{\circ} 14^{\prime} 47^{\prime \prime} \mathrm{S}$ & $50^{\circ} 23^{\prime} 41^{\prime \prime} \mathrm{W}$ & \\
\hline & 150 & Represa de Três Irmãos & $20^{\circ} 54^{\prime} 02{ }^{\prime \prime} \mathrm{S}$ & $50^{\circ} 34^{\prime} 03^{\prime \prime} \mathrm{W}$ & \\
\hline & 151 & Lago SP-463 & $21^{\circ} 044^{\prime} 42 ” S$ & $50^{\circ} 27^{\prime} 50^{\prime \prime} \mathrm{W}$ & \\
\hline & 152 & Lago Marechal Rondon & $21^{\circ} 12^{\prime} 03^{\prime \prime} \mathrm{S}$ & $50^{\circ} 32^{\prime} 42^{\prime \prime} \mathrm{W}$ & \\
\hline & 153 & $\begin{array}{l}\text { Lago Marechal Rondon - } \\
\text { Km } 580\end{array}$ & $21^{\circ} 11^{\prime} 44^{\prime \prime} \mathrm{S}$ & $50^{\circ} 53^{\prime} 52^{\prime \prime} \mathrm{W}$ & \\
\hline & 154 & Represa de Jupiá & $20^{\circ} 45^{\prime} 09^{\prime \prime} \mathrm{S}$ & $51^{\circ} 37^{\prime} 21^{\prime \prime} \mathrm{W}$ & \\
\hline & 155 & $\begin{array}{l}\text { Lago Marechal Rondon - } \\
\text { Km } 628\end{array}$ & $20^{\circ} 57^{\prime} 04^{\prime \prime} \mathrm{S}$ & $51^{\circ} 16^{\prime} 42^{\prime \prime} \mathrm{W}$ & \\
\hline \multirow[t]{11}{*}{20 - Aguapeí } & 156 & Lago urbano & $22^{\circ} 12^{\prime} 57^{\prime \prime} \mathrm{S}$ & $49^{\circ} 38^{\prime} 45^{\prime \prime} \mathrm{W}$ & BIOTA FAPESP \\
\hline & 157 & Lago Fazenda Santa Thereza & $22^{\circ} 12^{\prime} 12^{\prime \prime} \mathrm{S}$ & $49^{\circ} 43^{\prime} 50^{\prime \prime} \mathrm{W}$ & \\
\hline & 158 & Represa Municipal Tupã & $21^{\circ} 51^{\prime} 45^{\prime \prime} \mathrm{S}$ & $50^{\circ} 32^{\prime} 10^{\prime \prime} \mathrm{W}$ & \\
\hline & 159 & Lago do Country Clube & $21^{\circ} 56^{\prime} 48^{\prime \prime} \mathrm{S}$ & $50^{\circ} 25^{\prime} 12^{\prime} \mathrm{W}$ & \\
\hline & 160 & Lago marginal - Rio Aguapeí & $21^{\circ} 42^{\prime} 24^{\prime \prime} \mathrm{S}$ & $50^{\circ} 30^{\prime} 48^{\prime \prime} \mathrm{W}$ & \\
\hline & 161 & $\begin{array}{l}\text { Lago da Rodovia } \\
\text { Assis Chateaubriand }\end{array}$ & $21^{\circ} 41^{\prime} 01^{\prime \prime} \mathrm{S}$ & $50^{\circ} 36^{\prime} 35^{\prime \prime} \mathrm{W}$ & \\
\hline & 162 & Rio Paraná & $21^{\circ} 20^{\prime} 53^{\prime \prime} \mathrm{S}$ & $51^{\circ} 51^{\prime} 450^{\prime \prime} \mathrm{W}$ & \\
\hline & 163 & Lagoa Central Rio Paraná & $21^{\circ} 17^{\prime} 45^{\prime \prime} \mathrm{S}$ & $51^{\circ} 51^{\prime} 02{ }^{\prime \prime} \mathrm{W}$ & \\
\hline & 164 & $\begin{array}{l}\text { Lagoa do Pau da Onça } \\
\text { Fazenda Bandeirante }\end{array}$ & $21^{\circ} 05^{\prime} 16^{\prime \prime} \mathrm{S}$ & $51^{\circ} 42^{\prime} 42^{\prime \prime} \mathrm{W}$ & \\
\hline & 165 & Lagoa do Marreco & $21^{\circ} 06^{\prime} 04^{\prime \prime} \mathrm{S}$ & $51^{\circ} 43^{\prime} 53^{\prime \prime} \mathrm{W}$ & \\
\hline & 166 & $\begin{array}{l}\text { Foz do Rio Aguapei } \\
\text { Fazenda Bandeirante }\end{array}$ & $21^{\circ} 03^{\prime} 04^{\prime \prime} \mathrm{S}$ & $51^{\circ} 45^{\prime} 58^{\prime \prime} \mathrm{W}$ & \\
\hline \multirow[t]{6}{*}{21 - Peixe } & 167 & Represa Municipal Cascata & & & BIOTA FAPESP \\
\hline & 168 & Represa Fazenda Três Rios & & & \\
\hline & 169 & Represa da Fazenda Jabuti & & & \\
\hline & 170 & $\begin{array}{l}\text { Lagoa marginal do } \\
\text { Rio do Peixe }\end{array}$ & & & \\
\hline & 171 & Lagoa Nascente do Rib. Claro & & & \\
\hline & 172 & Foz do Rio do Peixe & & & \\
\hline \multirow[t]{11}{*}{ Várias UGRHi } & 173 & Represa Cabuçu & & & Tundisi (1980) \\
\hline & 174 & Rio das Pedras & & & Tundisi (1980) \\
\hline & 175 & Represa Guarapiranga & & & Tundisi (1980) \\
\hline & 176 & Represa Juqueri & & & Tundisi (1980) \\
\hline & 177 & Complexo Paraibuna & & & Tundisi (1980) \\
\hline & 178 & Represa Batista & & & Tundisi (1980) \\
\hline & 179 & São José & & & Tundisi (1980) \\
\hline & 180 & Piraju & & & Tundisi (1980) \\
\hline & 181 & Rio Pari & & & Tundisi (1980) \\
\hline & 182 & Rio Novo & & & Tundisi (1980) \\
\hline & 183 & $\begin{array}{c}\text { Lagoas Marginais do } \\
\text { Rio Paraná }\end{array}$ & & & Sendacz (1993) \\
\hline
\end{tabular}


Tabela 2. Continuação...

\begin{tabular}{|c|c|c|c|c|c|}
\hline UGRHi & $\begin{array}{c}\text { Código dos } \\
\text { corpos d'água }\end{array}$ & Corpos d'água & Latitude & Longitude & Projeto ou autor \\
\hline & 184 & Lagoa Dourada & & & $\begin{array}{l}\text { Talamoni (1995), } \\
\text { Melão (1997) }\end{array}$ \\
\hline & 185 & Represa do Monjolinho & $22^{\circ} 00^{\prime} \mathrm{S}$ & $47^{\circ} 54^{\prime} \mathrm{W}$ & $\begin{array}{l}\text { Nogueira (1990), } \\
\text { Okano (1995) }\end{array}$ \\
\hline & 186 & $\begin{array}{l}\text { Viveiros de Aquicultura } \\
\text { na UFSCar }\end{array}$ & & & Pamplin (1994) \\
\hline & 187 & Lagoa Pedreira São Carlos & $20^{\circ} 23^{\prime} 52,8{ }^{\prime \prime} \mathrm{S}$ & $51^{\circ} 21^{\prime} 13,3 ” \mathrm{~W}$ & Talamoni (1995) \\
\hline & 188 & $\begin{array}{l}\text { Viveiros de Aquicultura } \\
\text { (CEPTA - Pirassununga) }\end{array}$ & $22^{\circ} 02^{\prime} \mathrm{S}$ & $47^{\circ} 30^{\prime} \mathrm{W}$ & Fregadolli (1996) \\
\hline & 189 & $\begin{array}{l}\text { Viveiros de Aquicultura do } \\
\text { CAUNESP, Jaboticabal }\end{array}$ & $21^{\circ} 15^{\prime} \mathrm{S}$ & $48^{\circ} 18^{\prime} \mathrm{W}$ & $\begin{array}{l}\text { Sipaúba-Tavares \& } \\
\text { Colus (1997) }\end{array}$ \\
\hline & 190 & Lago Monte Alegre & & & Arcifa et al. $(1992,1997)$ \\
\hline & 191 & Rio Piracicaba & $19^{\circ} 38^{\prime} 12^{\prime \prime S}$ & $43^{\circ} 15^{\prime} 40^{\prime \prime} \mathrm{W}$ & $\begin{array}{l}\text { Zanata (2000), } \\
\text { Pareschi (2004) }\end{array}$ \\
\hline & 192 & Represa de Barra Bonita & $22^{\circ} 90^{\prime} \mathrm{S}$ & $48^{\circ} 34^{\prime} \mathrm{W}$ & $\begin{array}{l}\text { Zanata (2005), } \\
\text { Pareschi (2004) }\end{array}$ \\
\hline & 193 & Represa de Bariri & $22^{\circ} 06^{\prime} \mathrm{S}$ & $48^{\circ} 45^{\prime} \mathrm{W}$ & $\begin{array}{l}\text { Zanata (2005), } \\
\text { Pareschi (2004) }\end{array}$ \\
\hline & 194 & Represa de Ibitinga & $21^{\circ} 45^{\prime} \mathrm{S}$ & $48^{\circ} 59^{\prime \prime} \mathrm{W}$ & $\begin{array}{l}\text { Zanata (2005), } \\
\text { Pareschi (2004) }\end{array}$ \\
\hline & 195 & Represa de Promissão & $21^{\circ} 18^{\prime} S$ & $49^{\circ} 47^{\prime} \mathrm{W}$ & $\begin{array}{l}\text { Zanata (2005), } \\
\text { Pareschi (2004) }\end{array}$ \\
\hline & 196 & Represa Nova Avanhandava & $21^{\circ} 17^{\prime} 42^{\prime} \mathrm{S}$ & $50^{\circ} 08^{\prime} 17^{\prime \prime} \mathrm{W}$ & $\begin{array}{l}\text { Zanata (2005), } \\
\text { Pareschi (2004) }\end{array}$ \\
\hline & 197 & Represa Três Irmãos & $20^{\circ} 39^{\prime} \mathrm{S}$ & $51^{\circ} 18^{\prime \prime} \mathrm{W}$ & $\begin{array}{l}\text { Zanata (2005), } \\
\text { Pareschi (2004) }\end{array}$ \\
\hline & 198 & $\begin{array}{c}\text { Tanques de Piscicultura do } \\
\text { Insituto de Pesca }\end{array}$ & $22^{\circ} 55^{\prime} 50^{\prime \prime} \mathrm{S}$ & $45^{\circ} 27^{\prime} 22^{\prime \prime} \mathrm{W}$ & $\begin{array}{l}\text { Zanata (2005), } \\
\text { Pareschi (2004) }\end{array}$ \\
\hline & 199 & $\begin{array}{l}\text { Dez reservatórios do noroeste } \\
\text { do Estado de SP }\end{array}$ & $20^{\circ} 54^{\prime} 28^{\prime \prime} \mathrm{S}$ & $49^{\circ} 55^{\prime} 17^{\prime \prime} \mathrm{W}$ & Castilho-Noll et al. (2010) \\
\hline & 200 & Represa de Chavantes & $23^{\circ} 08^{\prime} 27^{\prime \prime} \mathrm{S}$ & $49^{\circ} 42^{\prime} 24^{\prime \prime} \mathrm{W}$ & Pomari (2010) \\
\hline & 201 & Represa do Broa - Mesocosmos & $22^{\circ} 15^{\prime} 10$ & $47^{\circ} 49^{\prime} 22$ & Gusmão (2004) \\
\hline & 202 & Lagoa do Camargo & & & $\begin{array}{l}\text { Panarelli (2004), } \\
\text { Mortali (2009) }\end{array}$ \\
\hline & 203 & Reservatório da UFSCar & $22^{\circ} 00^{\prime} \mathrm{S}$ & $47^{\circ} 54^{\prime} \mathrm{W}$ & Lucinda (2007) \\
\hline & 204 & Reservatório Chavantes, & $23^{\circ} 08^{\prime} 27^{\prime \prime} \mathrm{S}$ & $49^{\circ} 42^{\prime} 24^{\prime \prime} \mathrm{W}$ & $\begin{array}{l}\text { Sampaio et al. (2002), } \\
\text { Neves (2008) }\end{array}$ \\
\hline & 205 & Reservatório Salto Grande & $22^{\circ} 56^{\prime} 43^{\prime \prime} \mathrm{S}$ & $49^{\circ} 57^{\prime} 60^{\prime \prime \prime} \mathrm{W}$ & $\begin{array}{l}\text { Sampaio et al. (2002), } \\
\text { Neves (2008) }\end{array}$ \\
\hline & 207 & Resrevatórios de Jurumirim & $23^{\circ} 26^{\prime} 33^{\prime \prime} \mathrm{S}$ & $48^{\circ} 39^{\prime} 49^{\prime \prime} \mathrm{W}$ & Sampaio et al. (2002) \\
\hline & 208 & Reservatório de Piraju & $23^{\circ} 09^{\prime} 37^{\prime \prime} \mathrm{S}$ & $49^{\circ} 22^{\prime} 36^{\prime \prime} \mathrm{W}$ & Sampaio et al. (2002) \\
\hline & 209 & Rio Pari & & & Sampaio et al. (2002) \\
\hline & 210 & Rio Capivara & $22^{\circ} 54^{\prime} 01^{\prime} \mathrm{S}$ & $50^{\circ} 47^{\prime} 30^{\prime \prime} \mathrm{W}$ & Sampaio et al. (2002) \\
\hline & 211 & Lagoa Cavalos & & & Panarelli (2004) \\
\hline & 212 & Lagoa do Coqueiral & & & $\begin{array}{c}\text { Panarelli (2004), Nadai \& } \\
\text { Henry (2009) }\end{array}$ \\
\hline & 213 & Represa de Paraitinga & $23^{\circ} 24^{\prime} 44,6^{\prime \prime} \mathrm{S}$ & $45^{\circ} 35^{\prime} 28,4^{\prime \prime} \mathrm{W}$ & Nascimento (2008) \\
\hline & 214 & Represa de Rosana & $22^{\circ} 36^{\prime} \mathrm{S}$ & $52^{\circ} 50^{\prime} \mathrm{W}$ & Sartori (2008) \\
\hline
\end{tabular}

um número muito grande de pequenas lagoas ricas em vegetação. Acredita-se que para os Chydoridae, cladóceros habitantes da região litorânea dos lagos, futuros estudos que priorizem a região litorânea de pequenos lagos vegetados venham a ampliar a diversidade de espécies de cladóceros desta família. Para os Cladocera limnéticos considera- se que a ampliação de $15 \%$ obtida foi grande e que os resultados do projeto constituíram um grande avanço no conhecimento. Para os Chydoridae um grande avanço foi também obtido com a formação de recursos humanos e na cuidadosa identificação taxonômica que veio a esclarecer muitos registros duvidosos. Assim, consideramos 
que a cobertura geográfica poderá ainda ser ampliada, com focos mais específicos para alguns grupos. As informações já obtidas no Programa BIOTA/FAPESP permitirão que as amostragens sejam agora direcionadas para as unidades de gerenciamento onde estas espécies ocorreram (Tabela 2).

\section{Perspectivas de pesquisa em Cladocera para os próximos 10 anos}

Para ampliar o conhecimento taxonômico, ecológico e comportamental desse grupo taxonômico é imprescindível a formação de recursos humanos especializados nesse grupo zooplanctônico nos próximos 10 anos. É importante estender o estudo que foi realizado no Programa BIOTA/FAPESP para outras áreas do Brasil, estabelecendo uma rede de comunicação e troca de informações, através da formação de Grupos de Pesquisa com objetivos comuns em varias partes do Brasil manter o grupo por longo tempo para dar continuidade à pesquisa. Deverão ser ampliados em alguns temas principais, como a Revisão Taxonômica dos complexos de espécies, utilizando estudos morfológicos, ecológicos e de genética molecular; Bar-coding de espécies de Cladocera; Estudos de ciclo de vida da maioria das espécies, especialmente da família Chydoridae, ainda muito pouco conhecidos; Estudos aplicados de utilização de novas espécies de Cladocera em aquicultura; Estudos ecológicos voltados especificamente para a preservação de ecossistemas aquáticos ricos em espécies de Cladocera visando a preservação da biodiversidade em águas doces, incluindo o estudo ecológico das espécies e suas funções nas comunidades e ecossistemas.

\section{Agradecimentos}

À FAPESP pelo financiamento das pesquisas, ao CNPq pelas bolsas concedidas aos pesquisadores; ao Dr. Célio Wisniewski pelo auxílio na organização dos dados e às pós-graduandas Natália Felix Negreiros e Lidiane Cristina da Silva pela colaboração na formatação e na pesquisa e organização da revisão bibliográfica.

\section{Referências Bibliográficas}

ARCIFA, M.S., SILVA, L.H.S. \& SILVA, M.H.L. 1998. The planktonic community in a tropical Brazilian reservoir: composition, fluctuations and interactions. Rev. Brasil. Biol. 58(2):241-254.

ARCIFA, M.S., GOMES, E.A.T. \& MESCHIATTI, A.J. 1992. Composition and fluctuations of the zooplankton of a tropical Brazilian reservoir. Arch. Hydrobiol. 123(4):479-495.

BROOKS, J.L. 1959. Cladocera. In Freshwater biology (W.T. Edmondson, ed). 2nd ed. John Wiley \& Sons, Inc, New York, 1247p.

CASTILHO, P.M.J. 2005. Validação de ensaios ecotoxicológicos com organismos autóctones Daphnia laevis e Ceriodaphnia silvestrii. Dissertação de mestrado em Tecnologia Nuclear-Materiais, Instituto de Pesquisas Energéticas e Nucleares.

CASTILHO-NOLL, M.S.M., CÂMARA ,C.F., CHICONE, M.F. \& SHIBATA, E.H. 2010. Pelagic and littoral cladocerans (Crustacea, Anomopoda and Ctenopoda) from reservoirs of the Northwest of São Paulo State, Brazil. Biota Neotrop.: link http//www.biotaneotropica.org.br/v10n1/en/abstract (último acesso em 20/10/2010).

CHOUERI, R.B. 2004. Influência da matéria orgânica dissolvida na toxicidade, transferência e bioacumulação de cobre por uma espécie de Cladocera. Dissertação de mestrado em Ecologia e Recursos Naturais, Universidade Federal de São Carlos, São Carlos.

DUMONT, H.J. \& SILVA-BRIANO M. 1998. A reclassification of the anomopod families Macrothricidae and Chydoridae, with the creation of a new suborder, the Radopoda (Crustacea: Branchiopoda). Hydrobiol. 384:119-149. http://dx.doi.org/10.1023/A:1003259630312

ELÍAS-GUTIÉRREZ, M. \& VARELA, C. 2009. An annotated checklist of the Cladocera of Cuba. Crustaceana 82(11):1353-1364. http://dx.doi.or $\mathrm{g} / 10.1163 / 001121609 \mathrm{X} 12487811051426$
ELMOOR-LOUREIRO, L.M.A. 2004. Phylogenetic relationships among families of the order Anomopoda (Crustacea, Branchiopoda, Cladocera). Zootaxa 760:1-26.

FORRÓ, L., KOROVCHINSKY, N.M., KOTOV, A. \& PETRUSEK, A. 2008. Global diversity of cladocerans (Cladocera; Crustacea) in freshwater. In Freshwater animal diversity assessment (E.V. Balian, C. Lévêque, H. Segers \& K. Martens). Developmnts in Hydrobiology, 198, p.177-184.

FREGADOLLI, C.H. 1996. Efeito da disponibilidade de alimento e da predação po ninfas de Odonata no crescimento e sobrevivência de larvas de tambaqui, Colossoma macropomum (Cuvier, 1818). Tese de doutorado em Ecologia e Recursos Naturais, Universidade Federal de São Carlos, São Carlos.

FREITAS, E.C. 2009. Utilização da espécie nativa Pseudosida ramosa (Crustacea, Cladocera) como organismo-teste em estudos ecotoxicológicos. Dissertação de mestrado em Ecologia e Recursos Naturais, Universidade Federal de São Carlos, São Carlos.

FREITAS, E. C. \& ROCHA, O. 2006a. A influência da temperatura sobre o ciclo de vida de Pseudosida ramosa (Crustacea, Cladocera), uma espécie endêmica Neotropical. In Estudos integrados em ecossistemas. Estação Ecológica de Jataí (J.E. Santos, J.S.R. Pires \& L.E. Moschini, org.) EDUFSCar, São Carlos, p.421-429.

FREITAS, E.C \& ROCHA, O. 2006b. The life cycle of Pseudosida ramosa, Daday 1904, an endemic Neotropical cladoceran. Acta Limnol Bras 18(34):293-303.

FREITAS, E.C. \& ROCHA, O. 2011. Acute toxicity tests with the tropical cladoceran Pseudosida ramosa: the importance of using native species as test organisms. Arch Environ Contam Toxicol. 60:241-249. http://dx.doi. org/10.1007/s00244-010-9541-2

FREY, D.G. 1982. Questions concerning cosmopolitanism in Cladocera. Arch. Hydrobiol. 93:484-502.

FREY, D.G. 1987a. The taxonomy and biogeography of the Cladocera. Hydrobiologia p.145-17.

FREY, D.G. 1987b. The non-cosmopolitanism of chydorid Cladocera: implications for biogeography and evolution. In Crustacean biogeography (Crustacean issues 4) (R.H. Gore \& K.L. Heck, ed.). A.A.Balkema, London, p.353-402.

FREY, D.G. 1995. Changing attitudes towards chydorids anomopods since 1769. Hydrobiologia 307:43-55. http://dx.doi.org/10.1007/BF00031996

FRYER, G. 1995. Phylogeny and adaptive radiation within the Anomopoda: a preliminary exploration. Hydrobiologia 307:57-68.

FRYER, G. 1987. Morphology and the classification of the so-called Cladocera. Hydrobiologia 145:19-28. http://dx.doi.org/10.1007/BF02530261

GREEN, J. 1981. Cladocera. In Aquatic biota of tropical South America: 5-9 (S.H. Hurlbert, G. Rodriguez \& N.D. Santos, ed.). San Diego State University, San Diego, California, p.22-57.

GÜNTZEL, A.M., ROCHA, O., MATSUMURA-TUNDISI, T. 2003. Life cycle of Macrothrix flabelligera Smirnov, 1992 (Cladocera, Macrothricidae) recently reported for the Neotropical region. Hydrobiologia 490(1-3):87-92.

GUSMÃO, L.F.M. 2004. Efeitos do cobre e cromo na comunidade zooplanctônica: um estudo experimental em mesocosmos. Dissertação de mestrado em Ciências da Engenharia Ambiental, Universidade de São Paulo, São Carlos.

GUSSO, P.K. 2004. Contaminação alimentar e seus efeitos na bionomia de Ceriodaphnia cornuta (Cladocera, Daphnidae). Trabalho de conclusão de curso de graduação em Biologia, Universidade Federal do Paraná, Curitiba.

KOROVCHINSKY, N.M. 1996. How many species of Cladocera are there? Hydrobiologia 321:191-204. http://dx.doi.org/10.1007/BF00143750

KOROVCHINSKY, N.M. 2002. Description of two new species of Diaphanosoma Fischer, 1850 (Crustacea, Branchiopoda, Sididae) from the United States and Canada and species richness of the genus in North America. Hydrobiologia 489:45-54. http://dx.doi. org/10.1023/A:1023247024015 
KOROVCHINSKY, N.M. 2005. Two new species of Diaphanosoma Fischer, 1850 (Crustacea: Branchiopoda: Cladocera) from the United States. Int. Review Hydrobiol. 90:201-208. http://dx.doi.org/10.1002/ iroh. 200410748

KORINEK, V. 1981. Diaphanosoma birgei $\mathrm{n}$. sp (Crustacea, Cladocera), a new species from America and it's widely distribution species Diaphanosoma birgei ssp. Lacustris n ssp. Can. J. Zool. 59:1115-1121.

KORINEK, V. 2002. Cladocera. In A guide to tropical freshwater zooplankton: identification, ecology and impact fisheries (C.H. Fernando, ed.). Backhuys Publishers, Leiden, p.69-122.

LANSAC-TÔHA, F.A., BONECKER, C.C., VELHO, L.F.M. \& LIMA, A.F. 1997, Comunidade zooplanctônica. In A planície de inundação do alto rio Paraná: aspectos físicos, químicos, biológicos e sócio-econômicos (A.E.A.M. Vazzoler, A.A. Agostinho \& N.S. Hahn, ed.). EDUEM, Maringá, p.117-155.

LANSAC-TÔHA, F.A., BONECKER, C.C., VELHO, L.F.M., SIMÕES, N.R., DIAS, J.D., ALVES, G.M. \& TAKAHASHI, E.M. 2009. Biodiversity of zooplankton communities in the Upper Paraná River floodplain: interannual variation from long-term studies. Braz. J. Biol. 69(2):539-549. Suppl.

LUCINDA, I. 2007. Estudo de comunidades planctônicas (bacterioplâncton, nanoflagelados, fitoplâncton e zooplâncton) em um pequeno reservatório tropical - experimentos com mesocosmos. 2007. Tese de doutorado em Ecologia e Recursos Naturais, Universidade Federal de São Carlos, São Carlos.

MATSUMURA-TUNDISI, T. 1984. Ocurrence of species of the genus Daphnia in Brazil. Hydrobiologia 112:161-165. http://dx.doi.org/10.1007/ BF00008082

MELÃO, M.G.G. 1997. A comunidade planctônica (fitoplâncton e zooplâncton) e produtividade secundária do zooplâncton de um reservatório oligotrófico. Tese de doutorado em Ecologia e Recursos Naturais, Universidade Federal de São Carlos, São Carlos.

MELO, D.C. Avaliação da toxicidade da água proveniente do tratamento de resíduos químicos para a alga Pseudokirchneriella subcapitata e para o cladócero Ceriodaphnia silvestrii. 2009. Iniciação Científica.

NADAI, R., HENRY, R. 2009. Temporary fragmentation of a marginal lake and its effects on zooplankton community structure and organization. Braz. J. Biol. 69(3):819-835. http://dx.doi.org/10.1590/S151969842009000400009

NASCIMENTO, V.C. 2008. Aspectos do enchimento da represa Paraitinga, sistema produtor Alto Tietê: Zooplâncton e qualidade da água. Dissertação de mestrado em Saúde Pública, Universidade de São Paulo, São Paulo.

NEVES, G.P. 2008. Efeitos do tempo de residência, morfometria e estado trófico sobre as assembléias de microcrustáceos (Cladocera e Copepoda) dos reservatórios de Chavantes e Salto Grande (rio Paranapanema, SP/ PR). Dissertação de mestrado em Zoologia, Universidade Estadual Paulista, Botucatu.

NOGUEIRA, M.G. 1990. Dinâmica das populações planctônicas e fatores físico-químicos de um pequeno sistema artificial raso (represa do Monjolinho, São Carlos, SP). Dissertação de mestrado em Ciências da Engenharia Ambiental, Universidade de São Paulo, São Carlos.

NOGUEIRA, P.F.M. 2002. Consumo e influência de polissacarídeos excretados por Anabaena spiroides (Cyanophyceae) sobre os efeitos do cobre em Simocephalus serrulatus (Cladocera, Daphnidae). 2002. Dissertação de mestrado em Ecologia e Recursos Naturais, Universidade Federal de São Carlos, São Carlos.

NOGUEIRA, P.F.M., MELÃO, M.G.M., LOMBARDI, A.T. \& VIEIRA, A.A.H. 2005. The effects of Anabaena spiroides (Cyanophyceae) exopolysaccharide on copper toxicity to Simocephalus serrulatus (Cladocera, Daphnidae). Freshw. Biol. 50:1560-1567.

OKANO, W.Y. 1995. Análise da estrutura e dinâmica populacional da comunidade zooplanctônica de um reservatório artificial (Represa do Monjolinho, São Carlos-SP). Tese de doutorado em Ecologia e Recursos Naturais, Universidade Federal de São Carlos, São Carlos.
PAGGI, J.C. 1995. Crustacea Cladocera. In Ecosistemas de aguas continentales. Metodologías para su estudio (E.C. Lopretto \& G. Tell, ed.). Ediciones Sur, La Plata, p.909-951.

PAMPLIN, P.A.Z. 1994. Distribuição sazonal da comunidade zooplanctônica em tanques de piscicultura com diferenças no grau de trofia. Relatório Final de Iniciação Científica, CNPq. 35p.

PANARELLI, E.A. 2004. Flutuações mensais de comunidade zooplanctônica e dinâmica das populações de Cladocera em lagoas marginais, na região de transição Rio Paranapanema - Represa de Jurumirim (SP). Tese de doutorado em Ciências Biológicas, Universidade Estadual Paulista Júlio de Mesquita Filho, Botucatu.

PARESCHI, D.G. 2004. Caracterização da fauna de rotifera em área alagada construída para tratamento de esgoto: Piracicaba (SP). Dissertação de mestrado em Ciências da Engenharia Ambiental, Universidade de São Paulo, São Carlos.

POMARI, J. 2010. Efeitos da Tilapicultura em tanques-rede sobre as assembléias zoopalnctônicas do reservatório de Chavantes, rio Paranapanema (SP/PR). Tese de doutorado em Ciências Biológicas, Universidade Estadual Paulista Júlio de Mesquita Filho, Botucatu.

ROCHA, G.S. 2009. Efeito do alimento contaminado com cobre em parâmetros da história de vida e produção secundária do cladócero Daphnia laevis. Dissertação de mestrado em Ecologia e Recursos Naturais, Universidade Federal de São Carlos, São Carlos.

ROCHA, O. \& GUNTZEL, A.M. 1999. Crustacea Branchiopoda. In Invertebrados de Água Doce. (D. Ismael, W.C. Valenti, T. MatsumuraTundisi \& O. Rocha, ed.). Programa BIOTA/FAPESP, São Paulo, p.109-120.

ROCHA, O., WISNIEWSKI, M.J.S., GÜNTZEL, A. 2003. Relatório final sobre a diversidade dos Cladocera no Estado de São paulo. In Relatório final do projeto: diversidade de zooplâncton em relação à conservação e degradação dos ecossistemas aquáticos do estado de São Paulo (Processo) (T. Matsumura-Tundisi). Programa BIOTA/FAPESP, Fundação de Amparo à Pesquisa do Estado de São Paulo, São Paulo.

ROSA, G.A.B. 2003. Estudo taxonômico das espécies de Cladocera das famílias Moinidae e Sididae com ocorrência no estado da São Paulo e descrição do ciclo de vida de algumas das espécies. Monografia, Universidade Federal de São Carlos, São Carlos.

ROSA, G.A.B. 2008. Estudo dos efeitos do fármaco propranolol para Ceriodaphnia silvestrii (Cladocera, Crustacea) com ênfase em efeitos nas populações. Dissertação de mestrado em Tecnologia Nuclear, Universidade de São Paulo, São Paulo.

SANTOS-WISNIEWSKI, M.J., ROCHA, O., GUNTZEL, A.M. \& MATSUMURA-TUNDISI, T. 2006. Aspects of the life cycle of Chydorus pubescens Sars, 1901 (Cladocera, Chydoridae). Acta Limnol. Bras. 18:305-310.

SARTORI, L.P. 2008. Compartimentalização longitudinal do reservatório de Rosana (Rio Paranapanema, SP/PR) - Variáveis limnológicas e assembléias zooplanctônicas. Tese de doutorado em Ciências Biológicas, Universidade Estadual Paulista Júlio de Mesquita Filho, Botucatu.

SENDACZ, S. 1993. Estudo da comunidade zooplanctônica de lagoas marginais do rio Paraná Superior. Tese de doutorado, Universidade de São Paulo, São Paulo.

SERAFIM JUNIOR, M., LANSAC-TÔHA, F.A., PAGGI, J.C., VELHO, L.F.M. \& ROBERTSON, B. 2003. River-lagoon system of the upper Paraná River floodplain, with A new record for Brazil 2. Braz. J. Biol. 63(2):349-356. http://dx.doi.org/10.1590/S1519-69842003000200020

SINEV, A.Y. \& ELMOOR-LOUREIRO, L.M.A. 2010. Three new species of chydorid cladocerans of subfamily Aloninae (Branchipoda: Anomopoda: Chydoridae) from Brazil. Zootaxa 2390:1-25.

SIPAÚBA-TAVARES, L.H., COLUS, D. 1997. Estrutura da comunidade fitoplanctônica e zooplanctônica em dois viveiros de cultivo semiintensivo de peixes (Jaboticabal, São Paulo, Brasil). Bol. Lab. Hidrobiol. 10:51-64. 
TALAMONI, J.L.B. 1995. Estudo comparativo das comunidades planctônicas de lagos de diferentes graus de trofia e uma análise do efeito de Microcystis aeruginosa (Cyanophyceae) sobre algumas espécies de microcrustáceos. Tese de doutorado em Ecologia e Recursos Naturais, Universidade Federal de São Carlos, São Carlos.

TAYLOR, D.J., FINSTON, T.L. \& HEBERT, P.D.N. 1998. Biogeography of a widespread freshwater crustacean: pseudocongruence and cryptic endemism in the North American Daphnia laevis complex. Evolution 52:1648-1670. http://dx.doi.org/10.2307/2411338

TUNDISI, J.G., MATSUMURA TUNDISI, T., HENRY, R., ROCHA, O. \& HINO, K. 1988. Comparações do estado trófico de 23 reservatórios do Estado de São Paulo: eutrofização e manejo. In Limnologia e manejo de represas: série monografias em limnologia (J.G. Tundisi, ed.). EESC-USP/ CRHEA/ACIESP, tomo 1 , vol 1, 506p.
TUNDISI, J.G. 1980. Relatório final do projeto "Tipologia de represas do Estado de São Paulo". FAPESP (Processo 1978/1).

ZANATA, L.H. 2000. Heterogeneidade ambiental do reservatório de Salto Grande (Americana-SP) com ênfase na distribuição de Cladocera. Dissertação e mestrado em Ciências da Engenharia Ambiental, Universidade de São Paulo, São Carlos.

ZANATA, L.H. 2005. Distribuição das populações de Cladocera (Classe Crustácea) nos reservatórios do Médio e Baixo rio Tietê: uma análise espacial e temporal. Tese de doutorado em Ciências da Engenharia Ambiental, Universidade de São Paulo, São Carlos.

ZANATA, L.H., ESPINDOLA, E.L.G., ROCHA, O. \& PEREIRA, R.H.G. 2003. First record of Daphnia lumholtzi Sars, 1885, exotic cladoceran, in São Paulo state (Brazil). Rev. Bras. Biol. = Braz. J. Biol. 63(4):717720. PMid:15029383.

Recebido em 19/07/2010

Versão reformulada recebida em 14/10/2010

Publicado em 15/12/2010 


\section{Apêndice}

Apêndice 1. Referências complementares.

Appendix 1. Additional references.

ARCIFA, M.S. 1976. The planktonic Cladocera (Crustacea) and aspects of eutrophication of Americana Reservoir, Brasil. Bol. Zool. University of São Paulo 1:105-145.

ELMOOR-LOUREIRO, L.M.A. 1998. Branchiopoda. Freshwater Cladocera. In Catalogue of Crustacea of Brazil (P.S. Young, ed.). Museu Nacional, Rio de Janeiro, p.15-41.

ELMOOR-LOUREIRO, L.M.A., SANTOS-WISNIEWSKI, M.J. \& ROCHA, O. 2008. Redescription of Alonella lineolata Sars, 1901 (Chydoridae) and its translocation to the subfamily Aloninae. Poster Presentation, In 8th International Symposium on Cladocera, Aguascalientes, Mexico (Abstract Book, p.78).

ELMOOR-LOUREIRO, L.M.A., SANTOS-WISNIEWSKI, M.J. \& ROCHA, O. 2009. New records of Parvalona (Crustácea: Anomopoda: Chydoridae) from Brazil, with first description of the male. Rev. Brasil. Zool. 26(2):369-373.

HEBERT, P.D.N., WITT, J.D.S. \& ADAMOWICZ, S.J. 2003. Phylogeographical patterning in Daphnia ambigua: regional divergence and intercontinental cohesion. Limnol. Oceanogr. 48:261-268. http://dx.doi.org/10.4319/1o.2003.48.1.0261

FREY, D.G. 1986. The non-cosmopolitanism of chydorid Cladocera: implications for biogeography and evolution. In Crustacean biogeography (Crustacean issues 4) (R.H. Gore \& K.L. Heck, ed.). Balkema, Rotterdam, p.237-256.

KOTOV, A.A. \& ELMOOR-LOUREIRO, L.M.A. 2008. Revision of Ilyocryprus Sars, 1862 (Cladocera: Ilyocryptidae) of Brazil with description of two new subspecies. Zootaxa 1962:49-64.

MATSUMURA-TUNDISI, T. 2003. Relatório final do projeto: diversidade de zooplâncton em relação à conservação e degradação dos ecossistemas aquáticos do estado de São Paulo (Processo) Programa Biota FAPESP, Fundação de Amparo à Pesquisa do Estado de São Paulo.

MATSUMURA-TUNDISI, T. \& TUNDISI, J.G. 1976. Plankton studies in a lacustrine environment. Oecologia 25:265-270.

MATSUMURA-TUNDISI, T., RIETZLER, A. C. \& TUNDISI, J. G. 1989. Biomass (dry weight) and carbon content of plankton crustacea from Broa reservoir (São Carlos, SP, Brazil) and its fluctuation across one year. Hydrobiologia 179:229-236.

MATSUMURA TUNDISI, T., RIETZLER, A.C., ESPÍNDOLA, E.L.G. \& TUNDISI, J.G. 1990. Predation on Ceriodaphnia cornuta and Brachionus calyciflorus by two Mesocyclops species coexisting in Barra Bonita reservoir (SP, Brazil). Hydrobiologia 198:141-155.

MORTARI, R.C. 2009. Distribuição espaço-temporal de Cladocera (Crustácea, Branchiopoda) em uma lagoa subtropical lateral ao Rio Paranapanema (zona de desembocadura na Represa de Jurumirim-SP). 2009. Tese de doutorado em Ciências Biológicas, Universidade Estadual Paulista Júlio de Mesquita Filho, Botucatu.

NEGREIROS, N.F., ROJAS, N.E.T., ROCHA, O., SANTOS-WISNIEWSKI, M.J. 2009. Composition, diversity and short-term temporal fluctuations of zooplankton communities in fish culture ponds (Pindamonhangaba), SP. Braz. J. Biol. 69(3):785-794. http://dx.doi.org/10.1590/S1519-69842009000400005

OLIVEIRA, L.D. 2010. Estudo da estrutura da comunidade zooplanctônica e sua relação com as cianobactérias em três reservatórios do médio rio Tietê, SP. Dissertação de mestrado em Ciências da Engenharia Ambiental, Universidade de São Paulo, São Carlos.

ROCHA, O. \& MATSUMURA-TUNDISI, T. 1990. Growth rate, longevity and reproductive performance of Daphnia laevis Birge, D. gessneri Herbst and D. ambigua Scourfield in laboratory cultures. Rev. Brasil. Biol. 50:915-921.

ROCHA, O. \& SAMPAIO, A.V. 1991. Composição, caracterização e variação sazonal da comunidade zooplanctônica da Lagoa Dourada, bacia hidrográfica do Lobo. An. Sem. Reg. Ecol. 6:23-46.

ROCHA, O., MATSUMURA-TUNDISI, T., TUNDISI, T. \& FONSECA, C.P. 1990. Predation on and by pelagic turbellaria in some lakes in Brazil. Hydrobiologia 198:91-101.

ROCHA, O., MATSUMURA-TUNDISI, T. \& SAMPAIO, E.V. 1997. Phytoplankton and zooplankton community structure and production as related to trophic state in some Brazilian lakes and reservoirs. Verh. Internat. Verein. Limnol. 26:559-604.

ROCHA, O., RIETZLER, A., ESPÍNDOLA, E.G., MATSUMURA-TUNDISI, T. \& DUMONT, H.H. 1998. Diversity of fauna in sand dune lakes of Lençóis Maranhenses, Brazil. I: The zooplankton community. An. Acad. Bras. Ci. 70(4):719-726.

ROCHA, O. et al. http://www.biotaneotropica.org.br http://www.biotaneotropica.org.br/v11n1a/pt/abstract?inventory+bn0271101a2011. Biota Neotrop., vol. 11, no. 1a.

ROCHE, K.F., SAMPAIO, E.V., TEIXEIRA, D., MATSUMURA-TUNDISI, T. 1993. Impact of Holoshestes heterodon Eigenmann (Pisces:Characidae) on the plankton community of a subtropical reservoir: the importance of predation by Chaoborus larvae. Hydrobiologia 254:7-20.

SAMPAIO, E.V. 2002. Composição, abundância e diversidade das comunidades zooplanctônicas em reservatórios do sudeste do Brasil (Bacias dos Rios Parapanema e São Francisco). Tese de doutorado em Ecologia e Recursos Naturais, Universidade Federal de São Carlos, São Carlos.

SANTOS, R.M. 2010. Estrutura das comunidades fitoplanctônica e zooplanctônica, com ênfase na produção secundária do zooplâncton, e fatores ambientais relacionados nos reservatórios do baixo rio Tietê, SP. Dissertação de mestrado em Ecologia e Recursos Naturais, Universidade Federal de São Carlos, São Carlos.

SANTOS-WISNIEWSKI, M.J. 1998. Distribuição espacial e produção secundária da comunidade zooplanctônica do Reservatório de Barra Bonita, SP. Tese de doutorado em Ecologia e Recursos Naturais, Universidade Federal de São Carlos, São Carlos. 